Euskal ikerketen aldizkaria | Revue d'études basques |

Revista de estudios vascos | Basque studies review

$21 \mid 2018$

Numéro XXI

\title{
Sur les probables interférences phonologiques entre basque et gascon
}

\section{Ivaylo Burov}

\section{OpenEdition}

\section{Journals}

Édition électronique

URL : https://journals.openedition.org/lapurdum/3782

DOI : 10.4000/lapurdum.3782

ISSN : 1965-0655

Éditeur

IKER

\section{Référence électronique}

Ivaylo Burov, «Sur les probables interférences phonologiques entre basque et gascon », Lapurdum [En ligne], 21 | 2018, mis en ligne le 01 janvier 2018, consulté le 23 février 2022. URL : http:// journals.openedition.org/lapurdum/3782 ; DOI : https://doi.org/10.4000/lapurdum.3782

Creative Commons - Attribution - Pas d'Utilisation Commerciale - Pas de Modification 4.0 International - CC BY-NC-ND 4.0 


\title{
Sur les probables interférences phonologiques entre basque et gascon
}

\author{
Ivaylo Burov \\ Université de Sofia Saint Clément d'Ohrid, Département d'études \\ romanes \\ ivailobourov@hotmail.com
}

\begin{abstract}
Nous sommes particulièrement reconnaissant à Charles Videgain, Jasone Salaberria et Alain Viaut pour les commentaires enrichissants et les suggestions utiles sur cet article dont la problématique a été présentée lors d'une conférence-débat organisée par IKER le 19 novembre 2014 à Bayonne.
\end{abstract}

Le basque et le gascon sont deux expressions linguistiques sans aucun lien génétique mais dont la proximité géographique et les zones de bilinguisme ont favorisé des interpénétrations dans les domaines de la phonologie, de la morphosyntaxe et du lexique. Le présent article se propose d'examiner uniquement les interférences phonologiques entre ces deux langues, qui ont souvent été attribuées (et parfois à tort) à l'influence d'un substrat commun. Il apparaîtra notamment que les changements phonétiques considérés comme spécifiquement basco-gascons se retrouvent loin au-delà de la zone aquitano-pyrénéenne qui coïncide grosso modo avec l'aire de diffusion des deux idiomes. Dans un contexte de débats scientifiques où foisonnent toutes sortes de spéculations sur la diachronie et l'origine même du basque, ces constats remettent en cause les thèses d'influences substratiques strictement locales pour les changements en question. En effet, seule une analyse de leur motivation phonético-phonologique (rôle de la syllabe, de la coarticulation, de la lénition) peut expliquer pourquoi ils se sont réellement produits, ce qui détermine l'approche qui sera adoptée ici. Certes, ceci n'exclut pas la pertinence des facteurs extralinguistiques (historico-politiques, géographiques, ethnoculturels, etc.) qui pourraient avoir conditionné la permanence d'un fonds linguistique commun basco-aquitain et dans le respect de la tradition en phonologie diachronique la section introductive en fera brièvement état. 


\section{Les conditions historiques}

Le gascon est incontestablement le plus ibérique des dialectes de l'Hexagone et tient lieu avec le catalan de zone de transition entre gallo-roman et ibéro-roman plutôt que de véritable frontière linguistique. La situation géographique de l'Aquitaine au croisement d'une multitude de variétés linguistiques - français, occitan, castillan, catalan, aragonais, basque -, dont le gascon participe par certains traits et dans une mesure variable, a inspiré les chercheurs à établir toutes sortes de rapports entre l'originalité de l'idiome et les peuples, connus et inconnus, qui ont laissé leur empreinte sur sa zone de diffusion.

Même si l'importance du substrat aquitain, qui aurait pour beaucoup partie liée avec l'euskarien, a souvent été surestimée, il est incontestable que le latin s'est implanté en Aquitaine sur une couche préceltique et vraisemblablement préindoeuropéenne. On sait à ce sujet que les Gaulois ne se sont jamais établis en masses compactes dans le Midi et qu'ils n'ont formé qu'une aristocratie de pouvoir sur fond ligure à l'est et ibère à l'ouest (Lafont 1991, Bec 1995). Un précieux témoignage de Strabon du $\mathrm{I}^{\mathrm{er}}$ siècle av. J.-C. nous renseigne en effet que les Aquitains ressemblaient davantage aux tribus de la Péninsule ibérique qu'aux Gaulois voisins ${ }^{1}$. Dans un célèbre passage des Commentarii de bello gallico de Jules César, datant de la même époque, on apprend aussi que «Gallos ab Aquitanis Garumna flumen dividit». Ce constat rejoint l'observation de Tuaillon (1976) que la Garonne n'est pas seulement une frontière naturelle mais aussi une frontière linguistique plus que tout autre fleuve français. Plus loin dans ces Commentaires César reproche aux Aquitains d'être venus en aide à leurs frères de sang hispaniques dans la guerre sertoriane contre les Romains. Les Aquitains ne se sont pas pour autant ralliés aux Gaulois lors du soulèvement de Vercingétorix contre les armées de César (Baldinger 1958 : 279). Hirschfeld (1913 : 214) fait savoir également qu'ils ont demandé d'être administrativement séparés des Gaulois pendant qu'Auguste réorganisait l'administration des provinces mais ont dû toutefois leur concéder des terres entre la Garonne et la Loire.

Tous ces témoignages historiques montrent clairement que l'Aquitaine n'a jamais été celtisée que superficiellement, le latin avançant directement sur un fond préceltique. Cet état de fait trouve une répercussion au niveau du lexique et de la toponymie. Hubschmid (1954) a recensé dix fois plus de mots préindoeuropéens en Aquitaine et en Aragon que de mots celtiques. Rohlfs (1952), de son côté, dresse une carte des toponymes se terminant par le suffixe -ós, -osse (en Gascogne), -oz, -otze (dans le domaine basque) et -ués, -ueste (en Aragon), réflexe préindoeuropéen du lat. -ANUM et du gaul. -ACUM. Témoins de la profondeur de la colonisation gallo-romaine, ces deux derniers suffixes se font de plus en plus rares et cèdent

1 Baldinger (1958 : 281) observe que le nom même des Aquitani rappelle par son suffixe celui d'autres peuples de la Péninsule pyrénéenne comme les Lusitani, les Carpetani, les Lobetani, les Erdetani, les Turdetani, etc. 
progressivement la place à -ós à mesure qu'on franchit la Garonne et pénètre dans le domaine gascon.

Ce qui demeure encore incertain aujourd'hui c'est l'origine et l'appartenance ethnique des Aquitains de même que le lien entre leur langue et l'euskarien ou ancien basque. Baldinger (1958 : 279) s'interroge ainsi s'ils étaient «des Ibères ou des Ligures ou un mélange entre les deux ou encore autre chose », tout en passant en revue les thèses de Gimpera et de Rohlfs sur la question. Selon le premier, les Aquitains n'étaient pas un groupe homogène mais «un conglomerado de pueblos muy diversos». Le second affirme que vraisemblablement «le basque appartient à un groupe ethnique indigène pré-ibérique » sans exclure l'hypothèse qu'il s'identifierait avec la langue des anciens Aquitains, elle-même intermédiaire entre le basque et le gaulois ${ }^{2}$.

Quoi qu'il en soit de toutes ces hypothèses, il est certain que l'ancien basque était la langue d'une civilisation pyrénéenne très ancienne dont les liens génétiques avec l'ibère ont été niés après la découverte des bronzes de Botorrita, d'Ascoli et d'autres inscriptions antiques. Ces mêmes monuments semblent prouver toutefois que l'ibère et l'euskara n'étaient pas uniquement des langues voisines en contact mais qu'ils partageaient bon nombre d'affinités au niveau de la phonétique, de la morphologie, du lexique et de la toponymie ( $c f$. Orpustan 2009). On sait également que les Aquitains n'étaient décidément pas des Celtes mais on ignore dans quelle mesure leur langue était proche de l'ibère ou de l'euskara. S'appuyant sur des documents ibères déchiffrés (dont notamment le bronze de Botorrita), Videgain (2003) conclut que le basque était inclus dans une zone peuplée de Celtibères mais que les Pyrénées échappaient à leur emprise. Ces régions étaient habitées par des peuples fort mal connus dont les Vascons, locuteurs présumés de l'ancien basque.

Malgré les spéculations historiques et ethnolinguistiques, les chercheurs s'accordent généralement pour reconnaitre que le substrat préroman en Aquitaine a été foncièrement différent de celui dans le reste du domaine d'oc et à plus forte raison dans le domaine d'oïl. Mais les influences substratiques, quelle qu'ait été leur ampleur, n'ont pas pu être décisives à elles seules pour le sort du gascon, idiome particulièrement aberrant au sein du domaine occitan et perçu comme un «lengatge estranh » dans les Leys d'amor. Pour comprendre son caractère hétéroclite, il faut prendre aussi en considération le processus de romanisation et les diverses influences subies par le latin implanté en Aquitaine.

Contrairement à Bourciez (1936 : 6) qui croit à une romanisation « rapide et très complète » de l'Aquitaine, Baldinger (1958: 282) estime qu'elle a avancé très lentement et "qu'il a fallu des siècles pour assurer la victoire de la culture et de la langue romaines dans ce pays rebelle ». Il souligne en effet qu'une large zone pyrénéenne comprenant une partie de l'Aquitaine n'a été latinisée qu'entre le $\mathrm{VI}^{\mathrm{e}}$ et le $\mathrm{VII}^{\mathrm{e}}$ siècle. Quant à l'îlot bascophone, il a montré une résistance

2 Cités par Baldinger (1958 : 279-280). 
encore plus forte en préservant sa langue originelle. D'après Videgain (2003), d'accord sur ce point avec Michelena ${ }^{3}$, la basque se serait conservé parce que ses locuteurs étaient peu cultivés et moins développés culturellement que les Ibères. Ces derniers auraient perdu leur langue non seulement parce qu'ils habitaient un territoire plus vaste et plus accessible que celui du basque mais aussi parce que "leur développement culturel [...] les a rendus plus vulnérables à la romanisation ». Les bascophones, en revanche, représentaient une communauté plus fermée et une civilisation plus primitive, ce qui les a vraisemblablement laissés à l'abri d'influences linguistiques externes.

Oyharçabal (2005: 8) remet en cause la thèse de l'isolement géographique qui aurait préservé le basque de disparition. D'après lui, "la zone aquitano-ibérique n'a pas été au cours des derniers millénaires une zone d'accès difficile, mais au contraire une zone de passage et de contacts continuels avec [...] des civilisations aussi riches et diverses que celles des ibères, celtes, romains, germains, arabes... ». Il s'accorde avec Cavalli-Sforza (1994) sur le fait qu'au début de notre ère la zone d'extension du basque a été plus importante qu'aujourd'hui ( $c f$. Carte 1). Cela aurait facilité l'adoption des modes de vie, croyances et structures étatiques des populations ouest-européennes avec lesquelles les bascophones se sont retrouvés en contact.

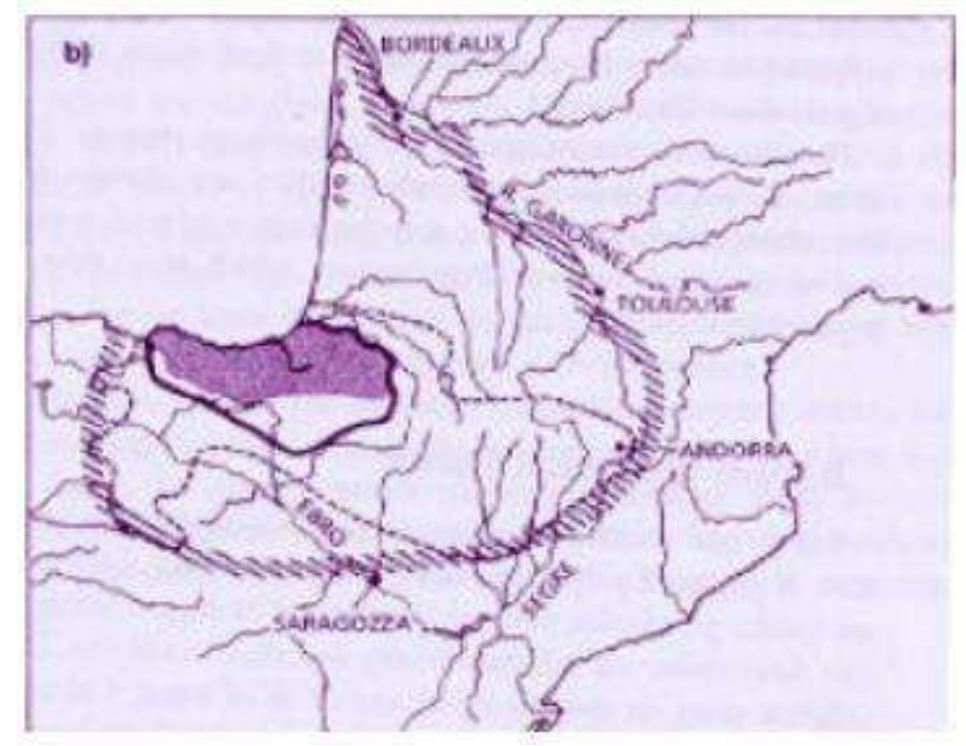

Carte 1 : En traits hachurés la limite de l'extension maximale du basque (Cavalli-Sforza 1994 : 322, Oyharçabal 2005 : 3)

Après la période de la romanisation, le latin de la nouvelle Provincia Novempopulana 4 a pu être orienté à plus d'une reprise vers la Péninsule pyrénéenne. Le royaume wisigothique de

3 Référence non citée.

4 Nom donné à l'ancienne Aquitaine vraisemblablement vers le début du III ${ }^{\mathrm{e}}$ siècle apr. J.-C. après la rupture administrative avec les Gaulois au nord de la Garonne (Baldinger 1958 : 282). 
Toulouse (466-507), quoiqu'il n'ait existé que pendant un demi-siècle ${ }^{5}$, a laissé par son extension jusqu'en Espagne une empreinte non seulement en Aquitaine mais aussi dans des régions marginales comme le Poitou, la Saintonge et l'Angoumois (Bec 1995 : 21). C'est probablement sous la poussée des Wisigoths, retirés en Espagne (royaume de Tolède) après la bataille de Vouillé, que les Vascons sont descendus des Pyrénées dans les plaines d'Aquitaine en lui imposant le nom de Vasconia vers l'an 600. S'il y a donc jamais eu des influences basques sur le latin de Gascogne, elles devraient s'être propagées et développées à une époque très lointaine, étant donné non seulement les faits historiques mais aussi le caractère très ancien des changements phonologiques dont il sera question en $\S 2$.

Bref, tout ce concours de circonstances historiques, et notamment le substrat aquitain et la fluidité des contacts avec le monde ibérique et bascophone, a pu faire basculer le gascon, qui est foncièrement un dialecte occitan, vers la Péninsule pyrénéenne. Le rôle des influences externes pour les changements à l'intérieur d'un système linguistique ne doit pas pour autant être surestimé parce que tout changement, qu'il ait été conditionné extérieurement ou non, a aussi nécessairement une motivation linguistique. La section suivante, qui ne sera consacrée qu'aux changements phonologiques communs au basque et au gascon, fera apparaître notamment que ceux-ci se retrouvent tous bien au-delà de la zone ibéro-aquitaine et le lecteur avisé pourrait juger par lui-même du bien-fondé des thèses substratistes.

\section{Les changements phonologiques dans la zone basco- gasconne}

Cette section sera réservée à l'analyse de six spécificités de la phonologie basque et gasconne, qui pourraient être dues à un contact de langues. Nous mettrons prioritairement l'accent sur la motivation phonétique et phonologique des changements examinés, en analysant ce qui se passe au niveau de l'articulation, de la perception et des structures syllabiques. Le recours à un modèle phonologique d'analyse particulier sera accompagné d'une brève introduction à son formalisme. Nous essaierons enfin d'identifier les aires d'expansion des changements afin de voir s'il s'agit réellement d'évolutions strictement locales ou non.

\section{1. Le traitement $\mathrm{du} / \mathrm{r} /$ initial}

Un des traits les plus spécifiques du gascon est sans doute le traitement du $r$ initial latin. Celuici a renforcé son articulation à tel point que le grand nombre de battements de la pointe de la langue contre la zone alvéolaire a provoqué un allongement sensible du temps d'émission de la vibrante. Cette espèce de géminée créée par exagération de l'effort articulatoire a donné à son tour naissance à une voyelle prothétique : $R \bar{I} V U>\operatorname{arriu}[$ arlriw] « ruisseau », *RACIMU >

5 Les Wisigoths commencent toutefois à s'installer en Aquitaine dès le début du V siècle. 


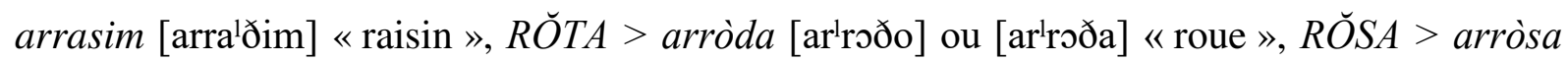
[ar'rozo] ou [arlroza] « rose » (Bec $1968: 173 ; 1995: 46)$.

L'origine du phénomène remonte à une époque assez lointaine ${ }^{6}$, car on en trouve des attestations dans des chartes du $\mathrm{X}^{\mathrm{e}}$ et du $\mathrm{XI}^{\mathrm{e}}$ siècle, où il affecte surtout des noms de personnes latinisés ou non : Arregemundo, Arreinaldo, Arroger, etc. (Ravier 1991 : 87, Baldinger 1958 : 260). Cette évolution n'est pas une originalité du gascon, mais un phénomène caractéristique des parlers ibériques et pyrénéens qu'il partage notamment avec l'aragonais et le basque. Ce dernier oppose à l'intervocalique une vibrante $/ \mathrm{r}$ :/ d'articulation prolongée et intense à une battue alvéolaire $/ \mathrm{r} /$. Les deux rhotiques ne se réalisent pas en début de mot et historiquement le $r$ initial des emprunts au latin ou à l'espagnol a été ramené au type vibrant et appuyé par un /e/ prothétique (variant dans certains items avec /a/) : ROTA > errota « roue », REGE > errege « roi », RATIONE > arrazoin « raison », respeto > errespeto, república > errepublika (Hualde 1991 : 12, Allières 1992 : 808).

Il est bien connu que l'espagnol allonge lui aussi le $r$ initial même si la langue standard n'est pas allée si loin dans son traitement que le gascon et l'aragonais : arag. arrier esp. reír « rire » (Ravier 1991 : 87). La prothèse dans ce contexte n'est pas cependant totalement inconnue de l'ancien castillan et la Carte 2 (reproduite d'après Baldinger 1958 : 259) identifie des endroits (indiqués par des points noirs) où elle est attestée entre le $\mathrm{X}^{\mathrm{e}}$ et le $\mathrm{XII}^{\mathrm{e}}$ siècle.

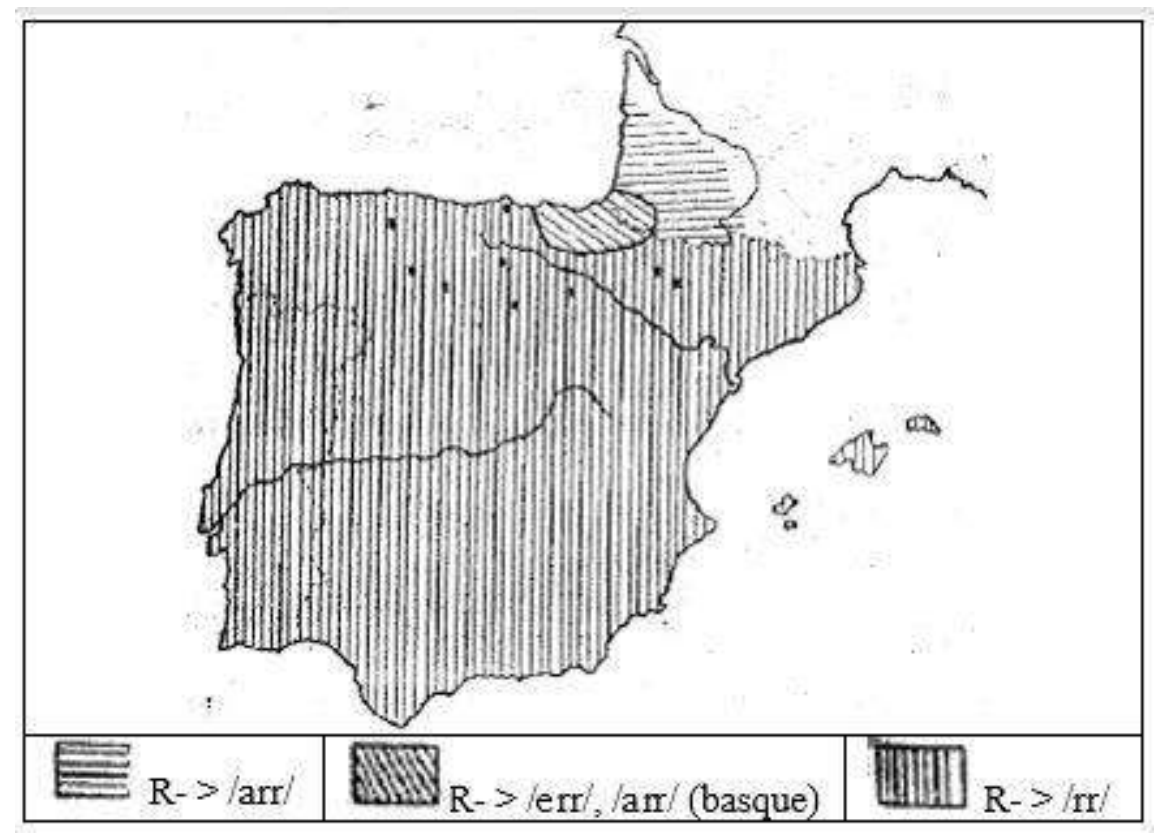

Carte 2 : Le traitement du /r/ initial (d'après Baldinger $1958: 259$ )

\footnotetext{
${ }^{6}$ Selon Millardet (1910), la prothèse serait apparue entre le $\mathrm{V}^{\mathrm{e}}$ et le VIII ${ }^{\mathrm{e}}$ siècle.
} 
Cet état de fait reflète apparemment une tendance articulatoire hispano-aquitaine que plusieurs linguistes ont été tentés de rattacher à des influences substratiques. Ravier (1991: 87) trouve cette hypothèse "d'autant plus séduisante que les noms indigènes des inscriptions latines de l'Aquitaine ignorent eux aussi r- initial (v. p. ex. ERIAPE ou ERRIAPE, divinité indigène abondamment attestée) ». A noter que la vibrante initiale était inconnue également de l'ibère (Orpustan 2009 : 6). Cependant, Bourciez (1923 : 456, 528, 579) signale que la prothèse se retrouve aussi en macédo-roumain $(R U B E U>$ aroybu «rouge », RIPA > arîpă « rive ») et marginalement en engadinois (domaine rhétique) et en émilien (domaine gallo-italien), ce qui jette l'ombre du doute sur la thèse substratiste.

Sur le plan phonologique l'apparition de la voyelle d'appui résulte de la difficulté de prononcer (ou de syllaber) un segment complexe dans une position marginale. Cette difficulté peut être mise en rapport avec une contrainte phonotactique du basque qui bannit systématiquement de la position initiale non seulement les groupes consonantiques hétérosyllabiques mais aussi des segments d'articulation complexe comme $/ K /, / \mathrm{h} /$, /r:/ ou les affriquées ${ }^{7}$ (Michelena $1954: 144$, Hualde 1991 : 12) qui ont normalement une durée d'émission plus longue ${ }^{8}$.

Même si le basque moderne accepte parfaitement les séquences d'une obstruante et d'une liquide /OL/, qui sont clairement tautosyllabiques non seulement dans les langues romanes, ses anciens emprunts au latin montrent qu'à ses origines il a essayé de s'en débarrasser par épenthèse (CRUCE > gurutze « croix », LIBRU > liburu « livre », CLETA > keleta « porte ») ou par aphérèse (PLUMA > luma «plume», FLORE > lore «fleur», GLORIA > loria «gloire») (Hualde 1991: 14, Orpustan 2009: 6). Ces faits peuvent être rapprochés des phénomènes de métathèse au contact de $/ \mathrm{r} /$ qui ont lieu avec une fascinante régularité non seulement en gascon mais aussi en aragonais et, d'une manière générale, en ibéro-roman. En voici quelques exemples recueillis par Baldinger (1958 : 246-247) dans des anciens textes de la région aquitaine : CAPRA > craba «chèvre», COMPRARE > crompar «acheter», INSTRUMENTU > esturmens « instrument », DORMIRE > dromir « dormir », PAUP $(E) R U$ > praube «pauvre », etc.

\footnotetext{
${ }^{7}$ A propos des affriquées, Hualde précise que l'apico-alvéolaire/tş/ et la lamino-dentale /tş/ sont rares en début de mot dans tous les dialectes. Quant à la prépalatale $/ \mathrm{t} /$, elle fait défaut dans beaucoup de dialectes orientaux sauf en biscaïen et en guipuscoan où historiquement un $/ \mathrm{g} /$ initial a été renforcé en $/ \mathrm{t} f /:$ labourd. xori $\sim$ bisc./guip. txori «oiseau ». D'autre part, en roncalais et en souletin, $/ \mathrm{t} f /$ est fréquent en position initiale dans des mots à valeur expressive : txakur/txákür « chien », txar « mauvais », etc. (Michelena 1954 : 147).

${ }^{8}$ On ne peut s'empêcher de faire un parallèle avec l'italien standard qui impose l'usage d'un article (défini ou indéfini) masculin à finale vocalique dès que le nom commence par un cluster hétérosyllabique ou par une consonne intrinsèquement longue comme $/ \mathrm{ts} /, / \mathrm{dz} /, / \mathrm{J} /, / \mathrm{K} / \mathrm{ou} / \mathrm{n} /:$ : uno/lo sciopero $[$ ['Jo:pero] « grève » un/il salto ['salto] «saut», uno/lo gnocco ['yokko] «petit pain» un/il nome ['no:me] «nom», uno/lo scudo ['sku:do] «écu » un/il grado ['gra:do] « degré » (Burov 2012, 2013a).
} 
Tout ce qui vient d'être dit témoigne d'une véritable difficulté de réalisation et de positionnement de $/ \mathrm{r} /$ due au faible gabarit des attaques syllabiques en gascon et surtout en basque. Dans cet ordre d'idées, l'apparition de la prothèse dans ces deux variétés découle d'une stratégie qui consiste à munir un segment initial extrasyllabique (représenté par la première moitié de la géminée /r:/) d'un noyau vocalique.

En Phonologie tridimensionnelle (Encrevé 1988), rejeton de la Phonologie autosegmentale (Goldsmith 1979), les géminées sont représentées comme des segments uniques associés à deux positions temporelles (1a). Mais comme une géminée est nécessairement à cheval sur deux syllabes successives, ces mêmes positions seront dominées par des composants syllabiques différents : $c f$. it. fatto « fait » $(1 \mathrm{~b})$ :
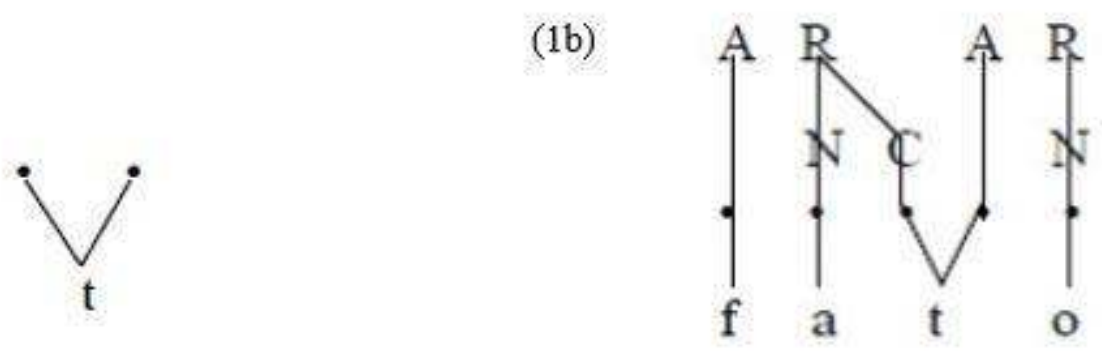

Dans ce modèle, la syllabe est conçue comme un complexe hiérarchisé à deux composants majeurs : Attaque (A) et Rime (R), cette dernière renfermant à son tour un segment syllabique appelé Noyau $(\mathrm{N})$ et facultativement une partie implosive appelée Coda $(\mathrm{C})$. La représentation (1b) fait apparaître aussi que composants syllabiques et segments ne s'associent pas directement mais se rejoignent en un point d'intersection commun appelé squelette. Innovation de la Phonologie autosegmentale, celui-ci représente une suite de positions pures dépourvues de tout contenu phonologique. Les positions squelettiques sont en effet des unités de temps qui illustrent la dimension chronématique de la parole et les rapports qu'entretiennent les segments successifs sur le plan syntagmatique. Elles s'associent à la fois avec les segments et les composants syllabiques. Comme l'indique Encrevé (1988), la première association permet aux segments de se réaliser phonétiquement, alors que la deuxième leur assure une interprétation prosodique au niveau de la syllabe.

Cela laisse entendre que, dans la représentation sous-jacente (ou lexicale) d'un mot, des segments peuvent rester non associés à aucun composant syllabique. Ce cas peut être illustré par un mot français comme porte où le /t/ final reste sous-spécifié syllabiquement au niveau lexical (2). Sa syllabation définitive ne se fera que postlexicalement et en fonction du contexte car les consonnes finales du français connaissent normalement un enchaînement (ou resyllabation) devant les mots commençant par une attaque nulle et une syllabation par défaut en coda dans les autres contextes : porte ouverte [ров.tu.veьt]. 

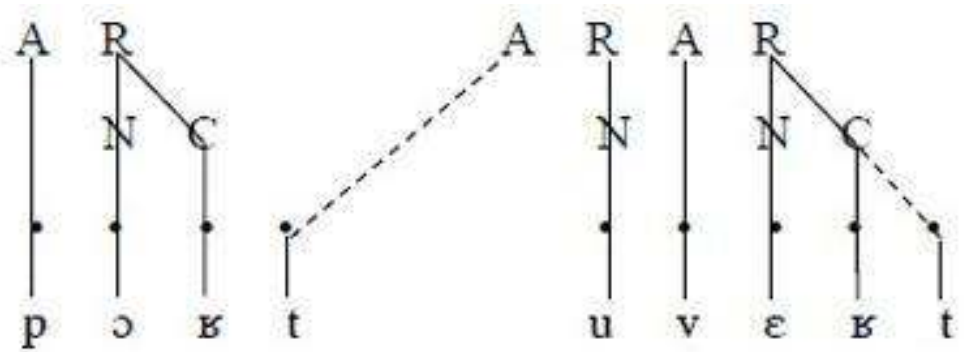

D'autre part, la représentation sous-jacente de l'adjectif ouverte, dont l'attaque initiale est nulle, montre que des composants syllabiques peuvent demeurer vides, c'est-à-dire non interprétés segmentalement. En effet, s'il y a des auteurs qui considèrent que le noyau est le seul élément obligatoirement rempli, Kaye \& Lowenstamm (1984) vont jusqu'à avancer une conception de la « syllabe minimale », selon laquelle celle-ci se présente obligatoirement sous la forme d'une structure binaire (AR), dont les deux composants peuvent rester nuls au niveau lexical. Ainsi, l'attaque doit être toujours structurellement présente, même dans les cas où elle ne domine pas de matériel segmental.

Cela étant, on peut poser qu'au moment où le /r/ latin de ROTA «roue » a intensifié son caractère vibrant en gascon, il y a eu passage d'une représentation sous-jacente à une autre :
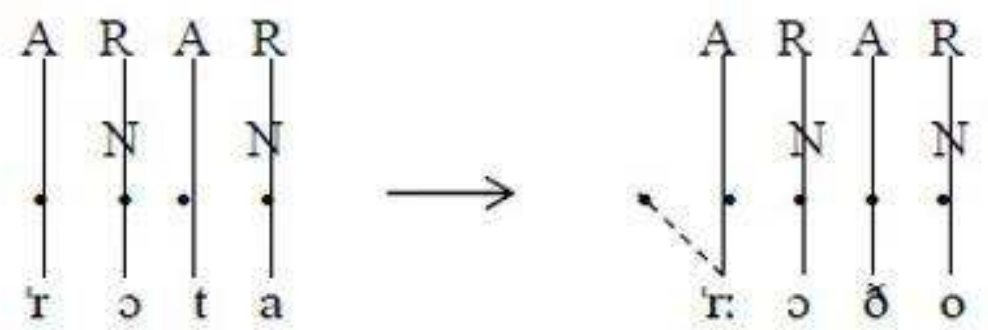

Dans un premier temps, $r$ s'est donc géminé par le dégagement d'une nouvelle position squelettique interprétée segmentalement comme une image miroir de la consonne de droite la plus proche. En même temps, cette position reste extrasyllabique (non interprétée prosodiquement) parce qu'aucune voyelle d'appui n'est encore disponible. Pour ce qui est du traitement ultérieur, il apparaîtra dans les lignes qui suivent que la prothèse n'est pas le seul moyen de rattacher cette position à un noyau syllabique.

En réalité, la prothèse de $a$ est née comme un phénomène de sandhi dépendant de la position du mot dans la chaîne segmentale. D'après Ronjat (1930 : 54), les formes avec prothèse n'apparaissaient à l'origine que dans les formes isolées ou placées après une finale consonantique, "ce qui ne se produisait pas quand $\mathrm{r}$ était entre voyelles dans un groupe étroitement lié tel que lo rei < (IL)LU REGE; puis il y aura eu normalisation de l'une ou de l'autre des formes alternées ». Cette normalisation par analogie se traduit par la perte de la fonction syllabique de la voyelle d'appui et s'explique par une contrainte d'uniformisation des représentations lexicales et graphiques en rapport étroit avec l'évolution de l'orthographe ( $c f$. Burov 2012, 2013a). 
Selon Bec (1968: 174), la théorie de Ronjat s'accorde parfaitement avec les particularités phonétiques du gascon moderne, à propos desquelles il ajoute :

\begin{abstract}
«L'effort articulatoire est, en effet, d'autant plus violent que le rr- se trouve à l'initiale absolue ou derrière une finale consonantique. Si l'on veut, par exemple, dans un groupe tel que : pétit rriw, conserver aux deux phonèmes du groupe disjoint : -t|rr- leur individualité articulatoire, une courte pause est nécessaire après le phonème implosif : il y a alors l'ébauche d'un point vocalique qui peut se développer davantage : pétit' rriw $>$ pétit ərriw > pétit arriw. La naissance de ce point vocalique suppose une articulation énergique de la consonne finale, ce qui s'admet parfaitement compte tenu des traits généraux du phonétisme gascon. Dans le cas d'une articulation moins énergique, la consonne finale s'assimile au rr- initial, augmentant ainsi le nombre des vibrements : pétir rriw. »
\end{abstract}

L'explication de Bec donne lieu de penser que l'assimilation régressive et la prothèse sont deux stratégies différentes de normalisation de la structure syllabique. La première consiste à projeter en position de coda un segment d'attaque (4a). En l'absence de contact entre les deux consonnes, à cause de la présence d'une pause réelle et/ou d'une frontière syntaxique trop forte, prend effet l'autre stratégie qui consiste à pourvoir la première partie de la géminée d'un noyau syllabique (4b).

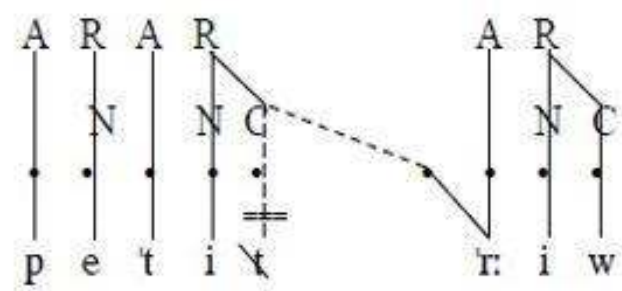

(4b)

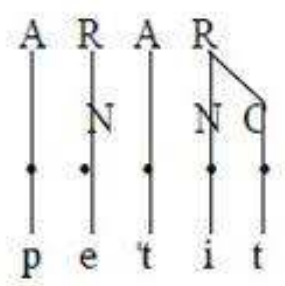

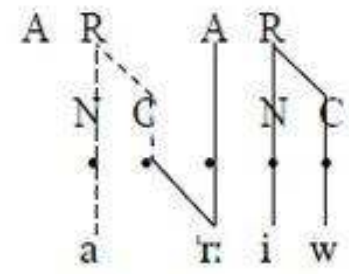

Contrairement à la plupart des variétés de gascon, où la prothèse a été lexicalisée au fil du temps, le luchonnais a préservé la distribution originelle $\mathrm{du} / \mathrm{a} / \mathrm{d}$ 'appui « qui reste un simple adjuvant articulatoire », pour reprendre l'expression de Bec (1968: 175). La prothèse n'y apparaît effectivement qu'après une pause ou une syllabe finale fermée, alors qu'après une syllabe ouverte le $r$ long est tout simplement resyllabé : $\breve{I L L A} R \breve{O T A}>$ [, era r'roðo] « la roue », UNA RŎSA > [jo r'rozo] « une rose ». Voici quelques autres exemples cités par l'auteur pour illustrer que le /a/ prothétique fonctionne exactement à la manière d'un schwa initial :

$$
\begin{aligned}
& \text { [, flus e r'ryßans] « fleurs et rubans » } \\
& {[\text {, luygo r'reygo] « longue rangée » }} \\
& {[\text {, sense , diðe rlrey] « sans rien dire » }}
\end{aligned}
$$

[, dyz arliws] « deux ruisseaux » $[\mathrm{pu}$, liðoz arl'rozos] « de jolies roses » [edz arralðim] « le raisin»

S'appuyant sur des données de Sarrieu, Bec donne aussi quelques exemples de maintien du $a$ prothétique après une syllabe ouverte, auquel cas le hiatus est cependant éliminé par effacement de la voyelle finale : [ym bulked de rlrozes] ou [ym bu'ked d arlrozes] « un bouquet de roses », [de rreln $\left.\varepsilon \int \mathrm{e}\right]$ ou [d arrelnefe] « de renaitre ». Ces alternances font penser que le /a/ d'appui est présent sous-jacemment sous la forme d'une voyelle flottante, sinon il serait impossible 
d'expliquer pourquoi la préposition de perd sa voyelle. Burov (2013a : 327) soutient que ces chutes ne peuvent découler que de la rencontre de deux voyelles flottantes dont l'une s'efface obligatoirement :
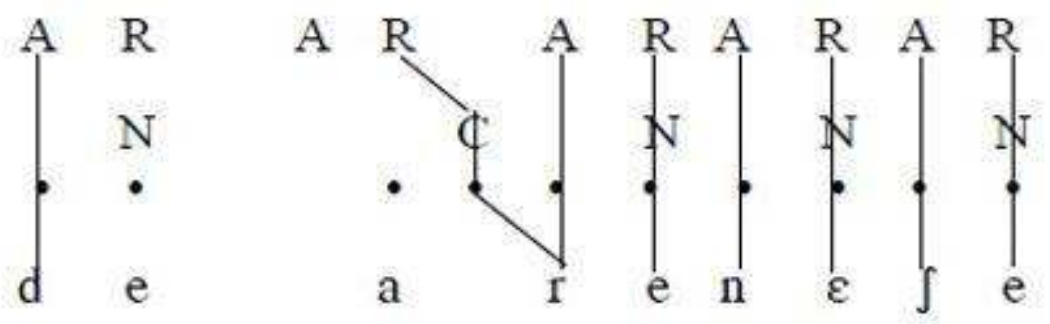

Dans ce modèle, les voyelles instables sont généralement encodées dans la représentation sousjacente par une $\bullet$ doublement flottante. Comme ces voyelles ne se réalisent pas nécessairement en surface, leur position ne sera reliée ni à la ligne segmentale, ni aux constituants syllabiques. En cas de réalisation, elle va s'ancrer dans les constituants disponibles, les éléments (autosegments) des différents niveaux restés non associés après l'application de toutes les opérations d'ancrage étant effacés :

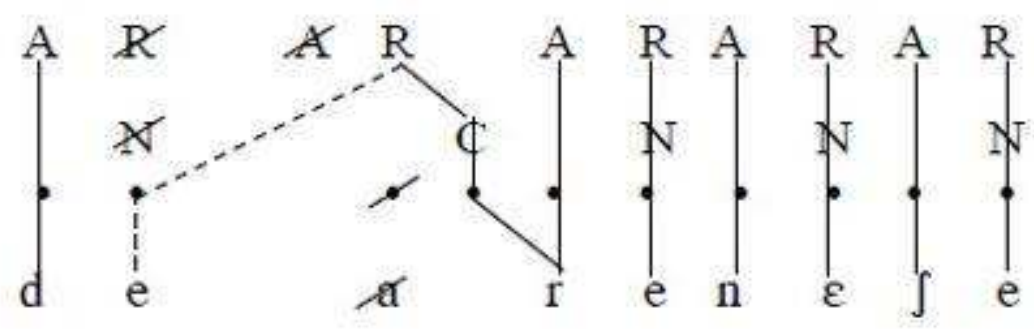

En somme, il ressort de ce qui a été dit précédemment que le /r/ initial s'est allongé dans toute la Péninsule ibérique et en Aquitaine. Contrairement à l'espagnol, où la prothèse est un phénomène diachroniquement isolé, le basque, le gascon et l'aragonais ont généralisé la voyelle adventice qui résulte de la nécessité de resyllaber le /r:/ géminé. Il existe toutefois des parlers gascons où le /a/ prothétique n'a pas été lexicalisé, préservant sa distribution originelle et sa fonction syllabique.

\section{2. Le passage de /f/ à /h/}

Le deuxième trait démarcatif du gascon par rapport aux autres parlers occitans est le traitement qu'il fait subir au $f$ latin : FËSTA > hèsta ['hesto] «fête », FILIA > hilha ['hiКo] «fille », FARINA > haria «farine », etc. Ravier (1991: 86) signale que dans certains parlers gascons (région bordelaise, Comminges, val d'Aran) l'aspiration qui apparaît à la place de la labiodentale tend à s'affaiblir davantage et à disparaître complètement : FOCU > [uk] «feu ». Les groupes secondaires $/ \mathrm{hr} /$ et $/ \mathrm{hl} /$ ont connu un sort différent selon les parlers, étant donné que dans ce cas l'affaiblissement a pour conséquence de transformer une séquence 
tautosyllabique en une séquence hétérosyllabique: /fr/, /fl/ $>/ \mathrm{h} . \mathrm{r} /$ et $/ \mathrm{h} .1 /{ }^{9}$. Ces produits s'accompagnent en effet de quatre stratégies de simplification dont la dernière est en interaction avec le traitement $\mathrm{du} / \mathrm{r} /$ initial.

La première stratégie consiste à insérer une voyelle épenthétique entre les deux éléments du

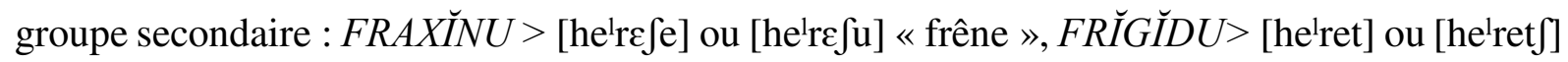
«froid », FLAMMA > [hallamo] ou [hallamu] «flamme », FLO $\bar{O} E>$ [hullu] «fleur ». Comme en témoignent ces exemples empruntés à Bec (1968 : 119-121), dans certains parlers gascons, l'épenthèse est doublée d'une harmonisation qui fait que la voyelle d'appui prend le timbre de la voyelle accentuée.

La deuxième stratégie est liée elle aussi à l'insertion d'une voyelle adventice mais devant le groupe de syllabation défectueuse : FLŌRE > [eh'lu] ou [es'llu], FLAMMA > [ehllama] ou [esllama], FLAGÉLLU > [esla'3zt] «fléau ». La prothèse n'apparaît que devant le groupe /hl/ et se caractérise par une énorme diversité des réalisations : [ehl-], [esl-], [ezl-], [ell-] ${ }^{10}$.

Les groupes initiaux $/ \mathrm{hr} /$ et $/ \mathrm{hl} /$ peuvent aussi rester intacts mais uniquement en liaison étroite

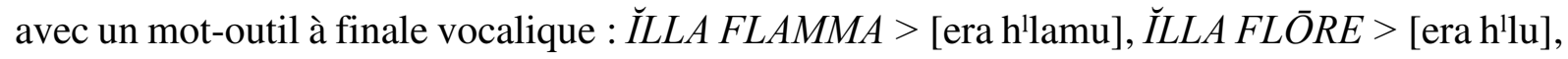
etc. Cela montre que les insertions vocaliques observées plus haut n'étaient à l'origine qu'un phénomène de sandhi né de la nécessité de réaliser une syllabation phonologiquement correcte au niveau de la phrase.

Enfin, l'évolution la plus poussée consiste à faire tomber l'élément initial du cluster hétérosyllabique. Connue d'un très grand nombre de parlers gascons, l'aphérèse affecte

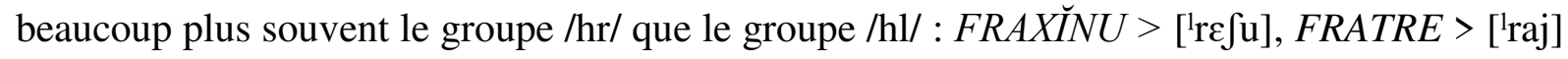
« frère », FORMATICU > [rulmadze] «fromage », FRUMENTU > [rulment] « froment », $F L A G \breve{E} L L U>$ [1a'3zt], FLŌORE > ['1u], FLAMMA > ['lama], etc. A en croire les observations de Bec (1968 : 119), "l’r initial, né de la réduction du groupe hr-, est en général articulé avec un seul vibrement, comme l'r intervocalique, sauf dans une partie du Gers où ce phénomène a été ramené au type commun de rr- initial (rrèšu). Il y a même des cas, rarissimes, de prothèse de a (arrèšu) ».

L'aspiration de /f/ est attestée également en espagnol (hija «fille», harina «farine », hilo «fil »), à ceci près que la labiodentale s'y maintient lorsqu'elle fait partie d'une attaque

\footnotetext{
9 Chomsky \& Halle (1973 : 127) classent /h/ et /2/ dans la catégorie des glides, c'est-à-dire parmi les segments de sonorité trop élevée pour que la formation d'une attaque complexe avec un segment subséquent soit possible.

10 Ce qui est curieux ici, c'est que l'aspirée, qui résulte de la débuccalisation de /f/, est ultérieurement interprétée comme une sifflante, à contre-courant de l'évolution $/ \mathrm{sC} />/ \mathrm{hC} /$ attestée en nord-occitan : escòla [ehkolo] « école ». Le passage de [h] à [s] s'explique certainement par la volonté de donner du corps à un segment menacé de disparition complète par sa substitution avec un segment suffisamment légitime en position de coda (Burov 2012, 2013a, b).
} 


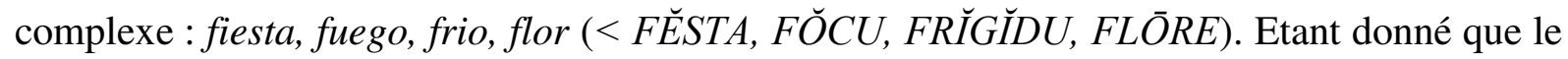
basque a éliminé cette consonne de ses emprunts au latin (FAVA > baba « fève », FESTA > besta, FILU > biru «fil», FORMA > borma «forme ») ${ }^{11}$ et à l'espagnol (Fernando > /pernando/, francés > /prantșes/ «français »), certains romanisants supposent une tendance articulatoire commune aux trois langues et sont enclins à rattacher le changement à un même substrat préroman.

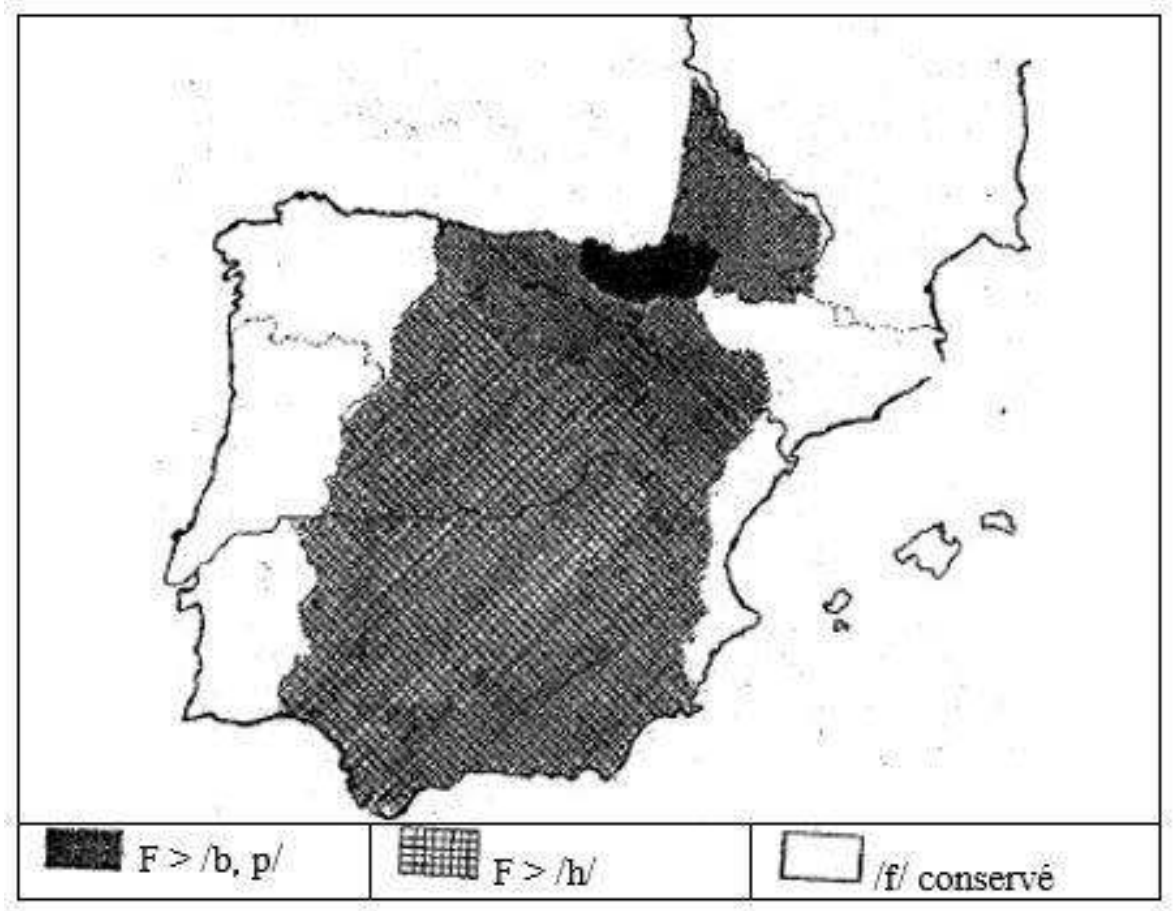

Carte 3 : Le traitement de /f/ (d'après Baldinger 1958 : 248)

Mais l'origine ibérique du phénomène a été remise en cause du fait qu'il se retrouve également dans certains dialectes du sud et du nord de l'Italie (calabrais, padouan, vénitien, lombard) : FILU > /'hilu/, FERRU > /her/ «fer», FOLIA > /'hoja/ «feuille », FEMINA > /'himmina/ «femelle», etc. (Rohlfs 1966 : 206). Des traces du même changement se retrouvent, quoique marginalement, jusqu'en macédo-roumain : FILIU >/lxiKu/ « fils » (Bourciez 1923 : 527). Aux prises avec la thèse substratiste, Gavel (1951 : 189) fait remarquer aussi que si le gascon s'était comporté avec la labiodentale latine comme le basque, celle-ci n'aurait pas dû se transformer en $/ \mathrm{h} /$, mais en /b/ comme en témoignent les exemples ci-dessus. En essayant de remonter aux sources du changement, Bec $\left(1968\right.$ : 117) affirme que la transformation panromane du I ${ }^{\mathrm{er}}$ siècle en vertu de laquelle les bilabiales latines $/ \phi /$ et $/ \mathrm{w} /$ se sont renforcées en labiodentales n'a tout

11 En ce qui concerne l'aboutissement du $F$ latin en basque, Gavel (1956:2), Allières (1992: 806) et Orpustan (2009: 6) notent l'existence d'une certaine variation dialectale : FAGU > bago, pago, phago, hago, fago « hêtre ». Le rétablissement d'une consonne sourde en début de mot, sans doute sous des influences romanes plus tardives, n'infirme pas toutefois la tendance générale au voisement des sourdes initiales du latin en basque (cf. § 2. 6) 
simplement pas eu lieu en Aquitaine et sur une partie de la Péninsule ibérique ${ }^{12}$. Au lieu de stabiliser son articulation par le travail des incisives supérieures, la labiale latine perd tous ses traits se référant à l'activité des organes de la cavité buccale et se transforme en une simple aspiration.

Le traitement différencié du $f$ latin (prononcé $/ \phi /$ à une époque archaïque) en basque et en gascon n'exclut pas pour autant l'existence d'une même tendance articulatoire sous-jacente. Plus concrètement, les fricatives non stridentes comme $/ \phi /, / \beta /, / \theta /, / \gamma /$, etc. sont perceptivement instables à cause du faible bruit de friction qui s'en dégage. Si elles ne basculent donc pas vers des réalisations occludées ou stridentes, le risque d'amuïssement est bien réel. Pour illustrer ceci il suffit de prendre comme exemple le traitement $\mathrm{du} / \mathrm{d} /$ intervocalique latin dans les dialectes sud- et nord-occitans respectivement. Par le stade /ð/, celui-ci a pris dans le Midi (y compris en gascon) une réalisation stridente /z/ et s'est maintenu : SUDARE > susar. Dans le nord, en revanche, la vulnérabilité perceptive de /ð/ a entraîné sa disparition complète : suar (cf. aussi fr. suer).

A cela on peut ajouter une autre évolution significative : celle de $[\beta]$ qui n'existe que comme allophone intervocalique de /b/ en basque ( $c f$. $\$ 2$. 5) et dans une partie du gascon (sud des Landes, Béarn, sud-ouest de la Bigorre) : / beße/, « boire », /lbiße/ « vivre » (Bec 1968 : 128, Ravier 1991 : 89). Dans le reste du domaine gascon, la fricative bilabiale non stridente, qui résulte de la confusion entre $B$ et $V$ latins, s'est affaiblie en [w] à l'intervocalique : BIBERE > bever/'bewe/, VIVERE > viver /'biwe/. Cet état de fait contraste avec la situation dans le complexe arverno-méditerranéen qui a abouti à une opposition entre /b/ [- continu, - strident] et $/ \mathrm{v} /$ [+ continu, + strident]. Allières (1992: 806) fait remarquer que la confusion entre $B$ et $V$ (prononcé /w/ à l'origine) a eu pour centre d'expansion la zone basco-gasconne qui n'a jamais connu /v/. C'est à partir de ce noyau primaire que le changement s'est ensuite propagé en occitan central et dans la zone hispanophone. En effet, le castillan n'éliminera la labiodentale sonore de son système consonantique qu'à partir du XVII ${ }^{\mathrm{e}}$ siècle.

D'un point de vue phonologique, il y a donc lieu de supposer que la mutation de $/ \phi /$ a été déclenchée par un problème de compatibilité entre les traits [+ continu] et [- strident]. Comme en témoignent les exemples des paragraphes précédents, une faible combinaison de traits distinctifs induit généralement deux types de changements : renforcement ou affaiblissement. Nous considérons donc que le gascon et partiellement l'espagnol diffèrent du basque et, d'une manière générale, des autres langues romanes quant à la stratégie adoptée pour remédier à la combinaison problématique. Le premier groupe de langues a opté pour l'affaiblissement $(/ \phi />$ $/ \mathrm{h} />(/ \varnothing /))$, alors que le second pour le renforcement. La basque se démarque cependant de la plupart des variétés de roman quant au type de renforcement opéré. Celles-ci ont modifié la

12 La Carte 3 illustre en effet que le passage /f/ > /h/ ne s'est pas accompli en portugais, en galicien, en aragonais, en asturien et en catalan. 
valeur originelle du trait [strident] $(/ \phi />/ f /)$, alors que celui-là la valeur originelle du trait [continu] $(/ \phi />/ \mathrm{p}, \mathrm{b} /)$. Ces mutations peuvent être schématisées de la façon suivante :

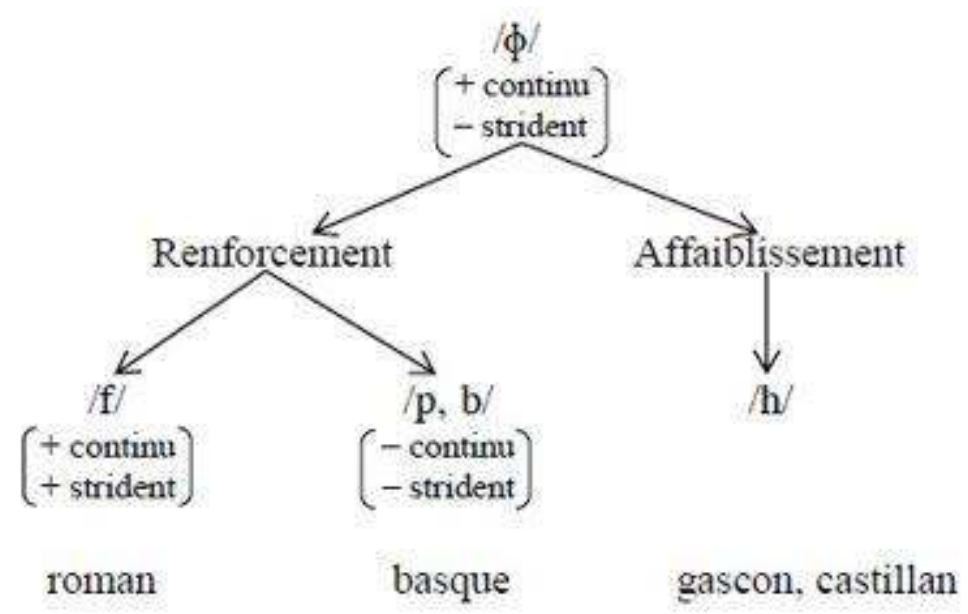

Dans un modèle phonologique où les éléments constitutifs des phonèmes sont des traits binaires, ces phénomènes de renforcement sont facilement formalisables. Qu'on adopte un modèle linéaire à la SPE (Chomsky \& Halle 1968), ou géométrique (Clements 1985, 1993, Clements \& Hume 1995, Sagey 1986, Halle, Vaux \& Wolfe 2000, etc.), la simple manipulation de la valeur + ou - des traits suffit pour rendre compte de l'évolution basco-romane. En Géométrie des traits, modèle qui repose sur l'organisation hiérarchique des traits et sur leur regroupement en nœuds différents, les phénomènes comparables à l'évolution hispanogasconne ci-dessus ont souvent été interprétés comme une débuccalisation ( $c f$., entre autres, Clements \& Hume 1995 : 271, Hualde 1991 : 131).

La version géométrique de Halle, Vaux \& Wolfe (2000), que nous avons adoptée et remaniée dans Burov (2013a), est connue sous le nom de Théorie articulatoire révisée (TAR). Elle est fondée sur le fonctionnement des six articulateurs de base, à savoir les lèvres, la lame de la langue, la masse de la langue, le voile du palais, la racine de la langue et le larynx. Chaque articulateur domine un petit ensemble de traits qui relèvent de son activité (9). Désignant les mouvements articulatoires réalisés à l'intérieur de la cavité buccale, les trois premiers articulateurs (Lèvres, Apex et Dorsum) sont logiquement subordonnés à un nœud supérieur Buccal, de même que les deux derniers (Radix et Larynx) sont subordonnés à Guttural en raison de leur proximité anatomique. Enfin, tout l'ensemble est gouverné par un nœud Racine (ou Pivot) qui exprime la cohérence du segment en tant qu'unité phonologique et contient les spécifications $[ \pm$ consonantique, \pm sonant], permettant de l'associer à une classe majeure (vocoïdes, sonantes ou obstruantes). 
(9)

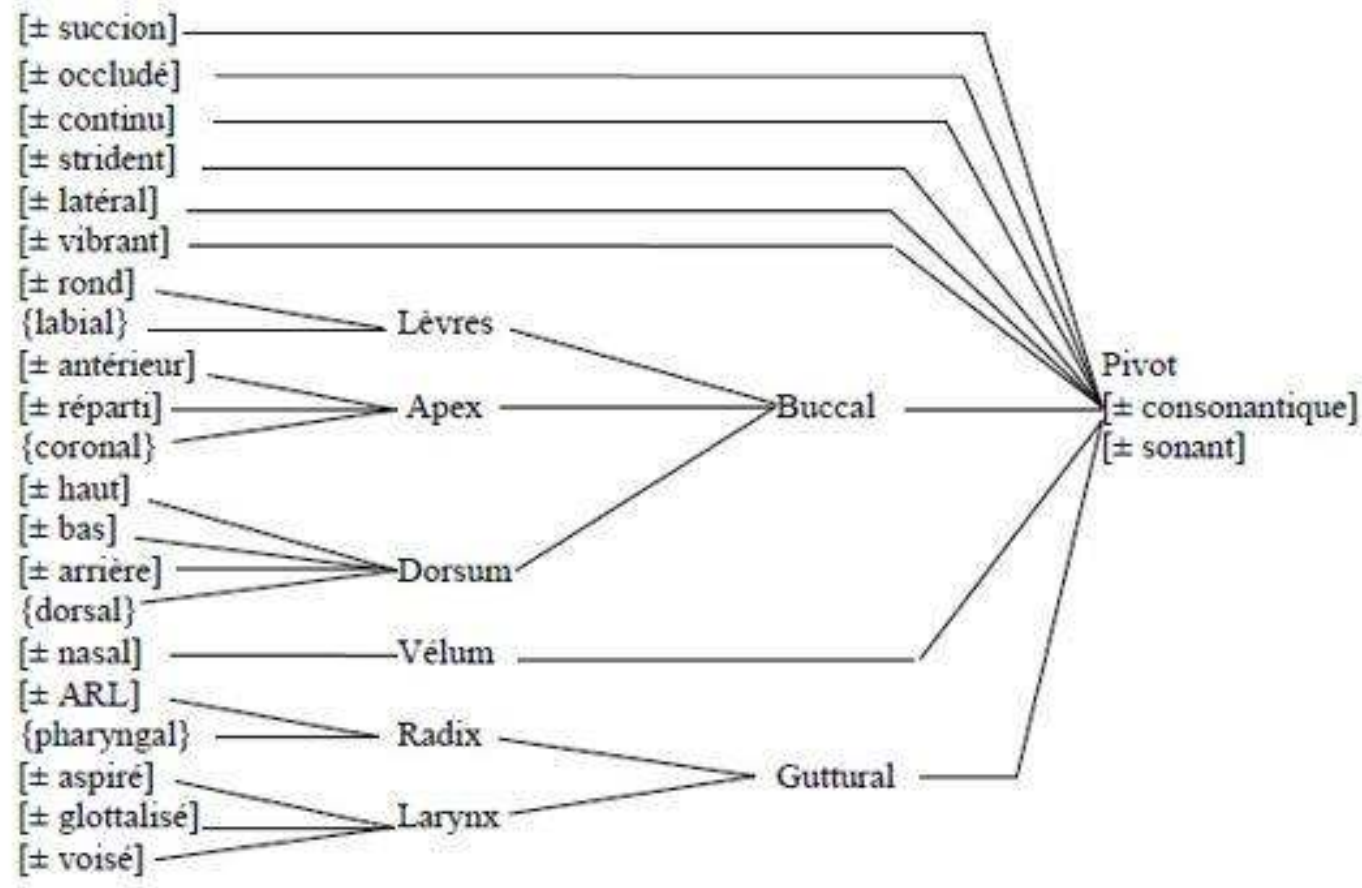

La TAR se distingue des autres modèles géométriques par la présence de traits unaires, notés entre accolades \{\} , indiquant l'articulateur de base du segment en question. Nous retenons que Lèvres, Apex, Dorsum et Radix dominent respectivement \{labial\}, \{coronal\}, \{dorsal\} et \{pharyngal\}, alors que Vélum et Larynx ne dominent pas des traits de lieu mais uniquement des traits binaires, notés entre crochets carrés [ ]. Enfin, certaines spécifications comme $[ \pm$ continu], $[ \pm$ latéral], $[ \pm$ strident], etc., qui caractérisent plusieurs types d'articulations, sont considérées articulator free est directement reliées au nœud Pivot.

Cela étant, le passage de $/ \phi /$ à $/ \mathrm{h} /$ sera interprété forcément comme une dissociation du nœud Buccal avec l'ensemble des traits pertinents qu'il domine. Comme les traits [continu] et [strident] n'en dépendent pas parce que directement reliés au nœud majeur Pivot, ils ne seront pas touchés. Il en résultera la fricative minimale $/ \mathrm{h} /$, segment sans lieu d'articulation identifié uniquement par le trait de mode d'articulation [+ continu $]^{13}$.

$13 \mathrm{La}$ thèse que $/ / /$ et $/ \mathrm{h} /$ sont dépourvus d'articulateur buccal et ne contiennent que des traits laryngiens est défendue par de nombreux phonologues (cf., entre autres, Sagey 1986 et Clements 1993). 
(10)

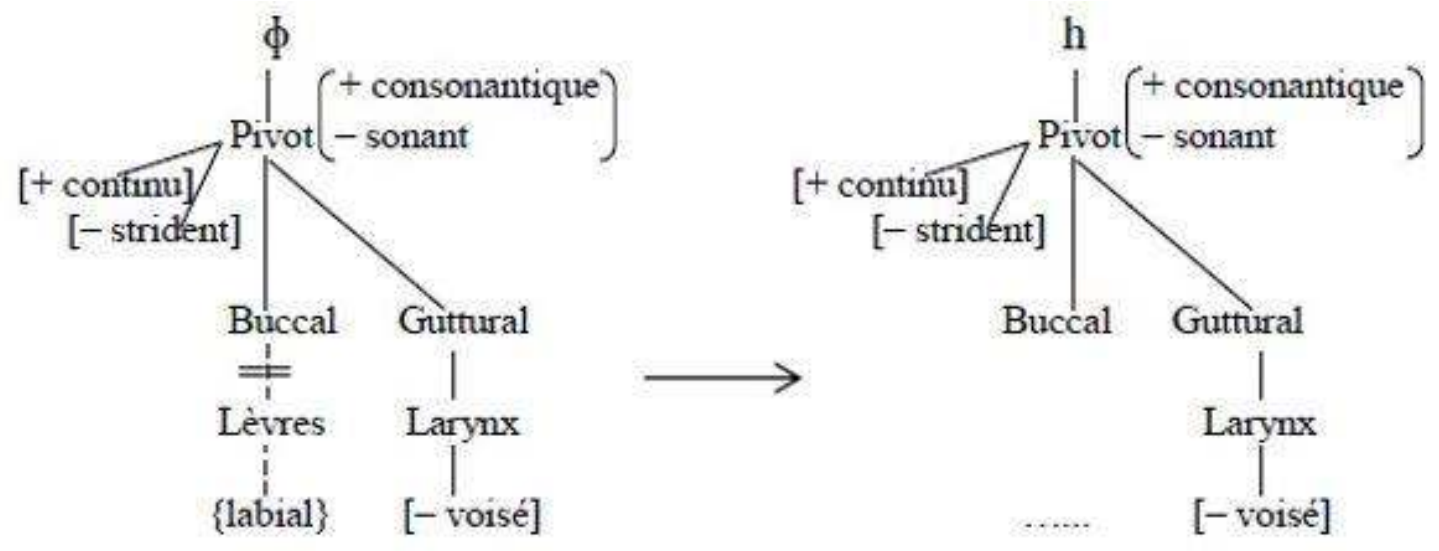

Même si le basque et le gascon modernes ont intégré /f/ à leurs inventaires consonantiques comme phonème d'emprunt tardif, on peut considérer qu'à une époque ancienne celui-ci a disparu également parce que non intégré à la corrélation de voisement. Comme il a été déjà dit, la labiodentale sonore / $\mathrm{v} /$ est absente des deux idiomes, alors que la fricative bilabiale sonore $/ \beta /$ n'y existe que comme allophone de $/ b /{ }^{14}$. Il est vrai qu'aujourd'hui le basque n'intègre à la corrélation de sonorité que les plosives, les affriquées et les fricatives étant toutes sourdes ${ }^{15}$. Martinet (1981) montre cependant que les fricatives sibilantes /s/, /S/ et /S/ dérivent vraisemblablement d'anciennes affriquées sonores homorganiques $/ \mathrm{dz} /$, /dz/ et $/ \mathrm{d} z /$, ce qui revient à dire qu'à une date ancienne toutes les obstruantes du basque étaient bien intégrées à la corrélation de voisement. Le tableau des oppositions entre sourdes et sonores ci-dessous est reproduit d'après Martinet (1981 : 65) à ceci près que nous avons transposé sa notation des consonnes en symboles de l'API :

\begin{tabular}{|c|c|c|c|c|c|c|c|}
\hline sourdes & $\mathrm{p}$ & $\mathrm{t}$ & ts & ts & t $\int$ & c & $\mathrm{k}$ \\
\hline sonores & b & d & $\perp$ & 1 & 1 & $f$ & $\mathrm{~g}$ \\
\hline
\end{tabular}

Selon les thèses fonctionnalistes et structuralistes, l'exclusion d'un phonème d'un système d'oppositions privatives régulières pourrait entraîner sa marginalisation et son évacuation du

14 Rappelons que dans la majeure partie de l'aire gasconne, l'allophone intervocalique de /b/ est [w] :BIBERE > /'bewe/, VIVERE > /'biwe/.

15 Ce n'est pas toutefois le cas du souletin qui possède dans son inventaire consonantique aussi bien des fricatives (/z/, /zl, /3/) que des affriquées sonores (/dzl, /dz/). Les paires minimales ou quasi-paires citées par Coyos (1999: 69) montrent l'indépendance phonémique des fricatives sonores par rapport aux sourdes homorganiques : /'sura/ « le bois de construction » /lzura/ «battre », /kasy/ «attention» /kazil/ «presque », / Jolraty/ «ébloui » /zolraty/ « sarclé ou critiqué ». 
système de la langue. La prégnance de la corrélation de sonorité pourrait donc avoir agi elle aussi dans le sens d'une élimination du $f$ latin.

Pour conclure, l'absence de /f/ des protosystèmes phonémiques du basque et du gascon est due probablement à une tendance générale à rejeter la combinaison des traits [+ continu] et [strident] que présentait le phonème latin $/ \phi /$. Sur le fond, les deux langues ne diffèrent que quant à la stratégie choisie pour réparer la combinaison perceptivement problématique. Enfin, les évolutions phonologiques examinées dans cette sous-section semblent montrer que la romanisation de l'Aquitaine ne devrait pas avoir été trop tardive, comme le veut Baldinger ( $c f$. $\S 1$ ), car en dehors de la Péninsule ibérique le renforcement panroman $\mathrm{du} / \phi /$ et $\mathrm{du} / \mathrm{w} / \mathrm{latins}$ s'est opéré vers le $\mathrm{I}^{\text {er }}$ siècle apr. J.-C.

\section{3. L'amuïssement $\mathrm{du} / \mathrm{n} /$ intervocalique}

Le gascon se démarque des autres dialectes occitans également par son traitement $\mathrm{du} / \mathrm{n} /$ intervocalique primaire : LUNA > lua /lyo/ «lune », FARINA > haria /halrio/ "farine », PLENA >//pleo/ «pleine ». Par ce fait il se rattache au basque qui a effectué le même changement dans ses emprunts au latin ainsi que dans des mots autochtones : CATENA > kate $(a)$ « chaîne », CORONA > koro $(a)$ « couronne », BALENA > bale $(a)$ « baleine », *ardano $>$ ardao > ardo « vin », * gaztane > gaztae > gazta « fromage ${ }^{16}$. Hualde (1991 : 84-85), à qui nous avons emprunté les exemples basques, fait remarquer que tout en disparaissant de certaines bases morphologiques, /n/ peut reparaître en dérivation :

$$
\begin{aligned}
& \text { kate }(a) \rightarrow \text { katen-begi «lien » gazta } \rightarrow \text { gaztan-bera « crème fromage » } \\
& \text { koro }(a) \rightarrow \text { koron-gile «faiseur de ardo } \rightarrow \text { ardan-degi «taverne » } \\
& \text { couronnes » }
\end{aligned}
$$

Hualde précise qu'à une époque ancienne les mots trisyllabiques comme katena, ardano, gaztane, etc. ont connu systématiquement une troncation de la voyelle finale lors des processus dérivationnels : ardano/ardan. Cette troncation étant antérieure à l'amuïssement de $/ \mathrm{n} /$, celuici n'a donc pas pu avoir lieu en l'absence du contexte intervocalique, d'où les alternances dans les mots composés en (12).

Comme en gallo-roman seul le /a/ final a persisté parmi les voyelles atones (et souvent sous une forme réduite), le traitement de /n/ devant les autres voyelles a été plus complexe en gascon. Ravier (1991 : 87) signale que le centre du domaine s'identifie avec une large zone vélarisante où les produits de $P A N E$ «pain » et $C A N E$ « chien » sont respectivement [pã $\tilde{a}^{\eta}$ et [kã $\tilde{a}^{\eta}$. Au nord de cette zone, la résonnance vélaire ne se fait pratiquement plus entendre et il en résulte une

16 Le - $a$ final a été parfois mal interprété comme la marque de l'article singulier postposé et éliminé de l'entrée lexicale (Hualde $1991: 85$ ). 
voyelle complètement nasalisée : [pã], [kã]. Au sud du domaine, en revanche, on constate une dénasalisation avec ou sans glissement du timbre de la voyelle originelle: [ka] ou [ko]. L'amuïssement $\mathrm{du} / \mathrm{n} /$ intervocalique est donc un changement très ancien, étant donné qu'il a dû s'accomplir avant la chute des voyelles finales. Il apparaît dès les premiers textes gascons et Ravier est enclin à le situer au VII ${ }^{\mathrm{e}}$ siècle.

Ce changement n'est pas confiné à l'aire basco-gasconne mais se retrouve également en galicien-portugais, comme en témoigne la Carte 4 tirée de Baldinger (1958 : 249) : LUNA > port. lua, gal. lúa. Rohlfs (1966: 312) en signale cependant des occurrences jusque dans le dialecte gallo-italien de Novara (Sicile) : /1ũa/, /fîlestra/ « fenêtre », /kaldẽa/ « chaine ».

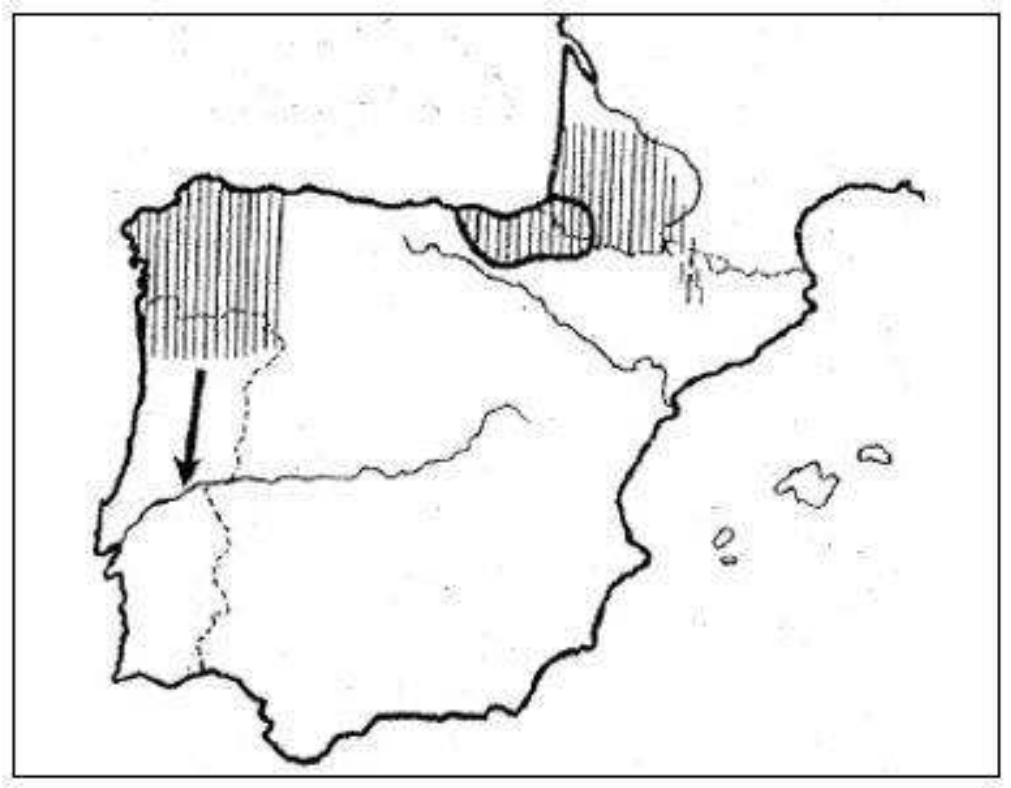

Carte 4 : Zones d'amuïssement du /n/ intervocalique (Baldinger 1958 :

249)

A la recherche d'une explication, Bec (1968:37-41) examine les faits de l'ancien portugais où le $n$ intervocalique a nasalisé la voyelle précédente (comme en sicilien) avant de disparaître : CORONA > corõa > coroa, LUNA > lũa > lua, BONA > bõa > boa «bonne ». Lorsque la nasale était précédée d'un /a/ accentué, celui-ci a préservé sa nasalité tout un absorbant un /a/ final atone : MANU > mão «main », LANA > la «laine», GERMANA > irma « sœur » ${ }^{17}$. En effet, l'abaissement du voile du palais est une conséquence automatique de la réalisation des voyelles basses, ce qui explique pourquoi celles-ci se nasalisent plus facilement que les autres (Barnes 2002 : 235).

17 A noter que le même changement est attesté à peu près partout en Aquitaine : LANA > / $1 a^{\eta} /$, ABELLANA > /awel $\tilde{a}^{\mathrm{p}} /$ « noisette » (Bec $\left.1968: 41\right)$. 
L'existence d'un stade intermédiaire de propagation de la nasalité aux voyelles contiguës ne fait aucun doute ni pour le basque, ni pour le gascon. Martinet (1981 : 63), Allières (1992: 804) et Coyos (1999: 78) notent à ce sujet que dans le dialecte souletin du basque la chute du /n/ intervocalique s'est accompagnée d'une nasalisation compensatoire des voyelles adjacentes qui n'est pas seulement régressive : ANATE > /ãlhãte/ « canard », HONORE > /ũlhũe/ « honneur », *BENE > /mẽhẽ/ «mince, maigre ». Cette persistance du trait de nasalité s'explique par son caractère saillant. Même après un affaiblissement extrême de l'obstruction buccale de /n/, il peut survivre dans le contexte environnant.

Michelena (1990 : 303-304) précise aussi que dans certains dialectes basques la disparition de la nasalité vocalique a entrainé le rétablissement de la consonne nasale. Celui-ci s'est produit notamment après une voyelle d'avant qui a pu faire basculer l'articulation consonantique vers la zone palatale : REGINA > a.-nav., guip., bisc. /er:egina/, soul. /er:elgina/, b.-nav., lab. /er:egina/ « reine »; CATENA > soul. /k ${ }^{\mathrm{h}}$ altina/ ; ARENA > soul. /hal(r)ina/ « sable ».

En essayant de reconstituer la diachronie du changement $L U N A>/ 1$ yo/ en gascon, Bec (1968 : 40) lui suppose les stades suivants : 1) la réalisation anticipée de la consonne nasale (en raison de la coarticulation) provoque un abaissement du velum pendant la phase vocalique et une légère nasalisation de $/ \mathrm{u} /:$ : [1una] > [1ũna ${ }^{18} ; 2$ ) à mesure que la nasalisation devient complète, $/ \mathrm{n} /$ s'affaiblit en appendice consonantique à résonnance vélaire : [1'ũna] > [1 $\left.{ }^{1} \tilde{u}^{\mathfrak{a} a}\right]^{19} ; 3$ ) l'articulation anticipée du /a/ final empêche la masse linguale de s'élever suffisamment, ce qui

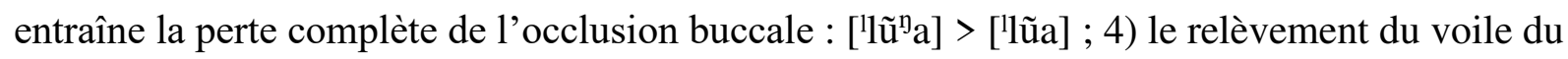
palais provoque la dénasalisation de la voyelle : [1'ũa] > ['lua].

Reste à savoir comment interpréter phonologiquement les évolutions liées à l'amuïssement de /n/ dans les théories présentées dans les deux sous-sections précédentes. Tout comme le passage de $/ \phi /$ à $/ \mathrm{h} /$, la chute de $/ \mathrm{n} /$ peut être conçue elle aussi comme une débuccalisation (dissociation du nœud Buccal avec ses traits dépendants). Cette dissociation n'affectera pas toutefois [+ nasal] qui, comme illustré en (9), est dominé par Velum, lui-même dépendant directement du nœud Pivot. Le caractère perceptivement saillant de [+ nasal], favorisant sa conservation même après la perte de l'obstruction orale, est ainsi adéquatement formalisé dans ce modèle.

18 La palatalisation occitane du $\bar{U}$ latin en /y/ paraît avoir été beaucoup plus tardive qu'en français et de toute façon bien postérieure à la chute du /n/ intervocalique ( $c f$. Bec 1999).

19 Cette phase vélaire est attestée dans certains dialectes liguriens et piémontais : ['lỹya] (Rohlfs 1966 : 312 ). 

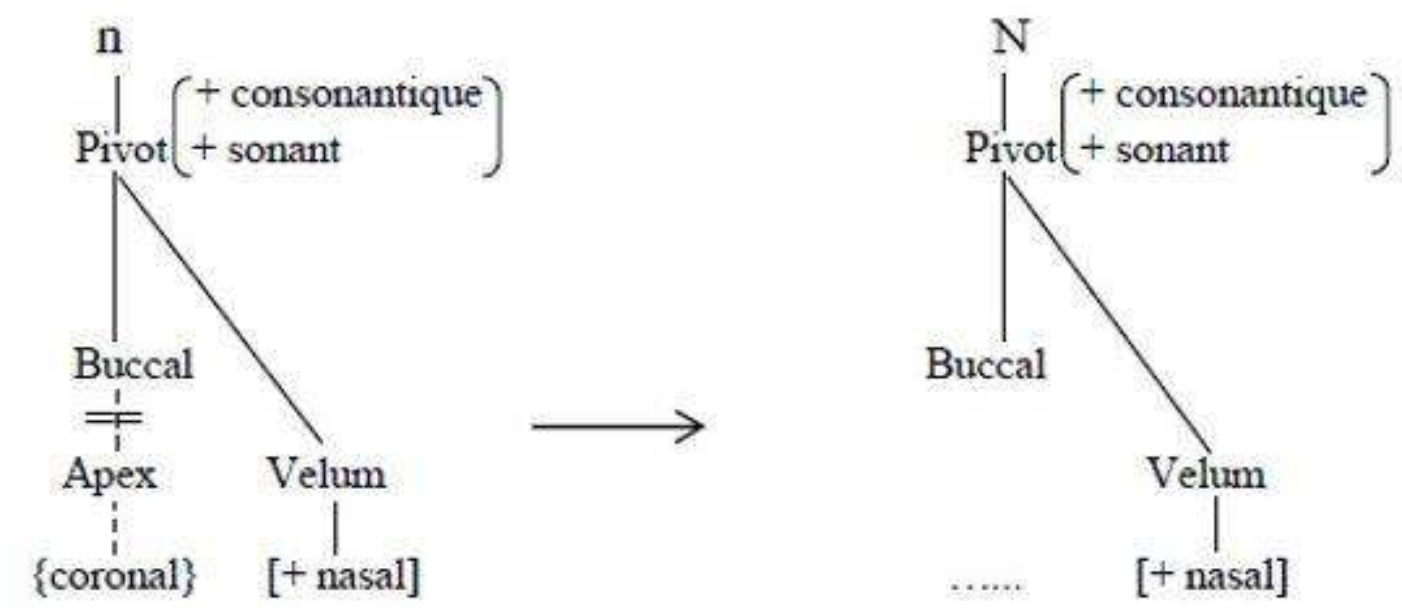

Il en résulte la nasale minimale / $\mathrm{N} /$, segment sans lieu d'articulation dans la cavité orale, identifié uniquement par le trait de nasalité. Pour se stabiliser segmentalement il s'associe par défaut à la voyelle précédente, laissant la position de l'attaque vide :

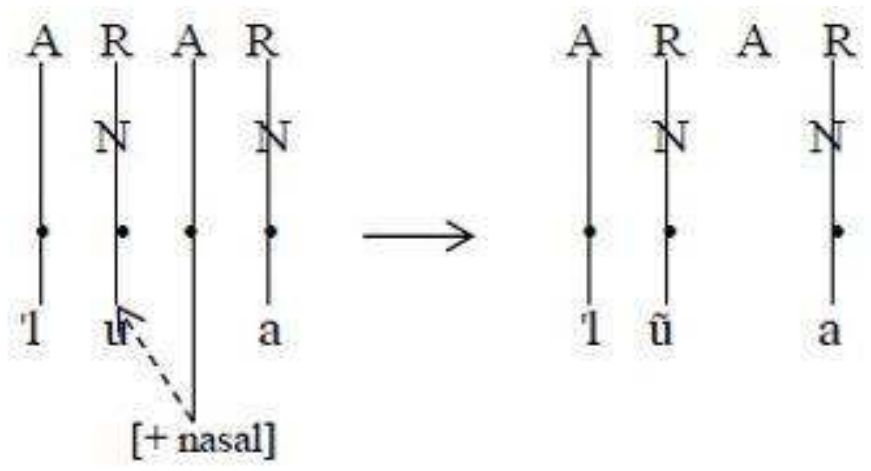

La dénasalisation subséquente s'explique de son côté par une faible compatibilité translinguistique de [+ nasal] avec [- consonantique] ou avec [+ continu]. La nasalité est en effet mieux perçue dans le cadre d'articulations discontinues $(/ \mathrm{m} /, / \mathrm{n} /, / \mathrm{n} /, / \mathrm{n} /$, etc.) qu'ailleurs. Cela rend les voyelles nasales très marquées phonétiquement et rares typologiquement, ce qui est un obstacle à leur phonologisation (intégration dans le système phonémique d'une langue).

En conclusion, le caractère très ancien de l'amuïssement $\mathrm{du} / \mathrm{n} /$ intervocalique dans la zone basco-gasconne est un argument en faveur des spéculations substratistes. D'un autre côté, celuici est le troisième changement examiné ici qui met en évidence des tendances articulatoires non locales. Cette fois-ci, ce sont le basque et le gascon qui, en accord avec le galicien-portugais, font dissonance avec l'espagnol. Aux dires de Baldinger (1958:251), ces deux zones séparées par les montagnes de la Cantabrie et des Asturies apparaissent «comme les piliers d'un pont détruit en son milieu». Même si aucun vestige de ce pont détruit n'a été découvert pour l'instant, les phases nasalisantes, vélarisantes et dénasalisantes, qui ont accompagné la chute de $/ \mathrm{n} /$, témoignent d'étonnantes similitudes entre les deux zones et certains parlers de l'Italie. 


\section{4. Le rhotacisme des latérales intervocaliques}

Le gascon est le seul dialecte occitan à avoir transformé la géminée intervocalique latine /1:/ en une battue alvéolaire : BELLA >/bero/ «belle », GALLINA>/galrio/ « poule », *APPELLARE $>$ /apelsa/ «appeler». Ceci le rapproche du basque qui est le seul idiome limitrophe à avoir opéré un changement semblable dans ses emprunts au latin : CAELU > zeru «ciel », ANGELU > aingeru « ange », VOLUNTATE > borondate « volonté ». Hualde (1991: 84), à qui nous avons emprunté les exemples basques, signale qu'à une date ancienne ce processus de rhotacisme a dû être très productif étant donné qu'il a produit des alternances morphophonémiques en synchronie entre /l/ et / $/$ / (15). Ces alternances ne peuvent manquer de rappeler celles examinées en (12) : *ardano > ardo mais ardan-degi. Dans les deux cas, la troncation de la voyelle finale a prévenu l'amuïssement de /n/ ou favorisé le maintien de /l/ par l'élimination du contexte intervocalique :

$$
\begin{aligned}
& \text { euskara «basque » } \rightarrow \text { euskal-liburu « livre basque » } \\
& \text { erdara «étranger » } \rightarrow \text { erdal-tzale «xénophile » } \\
& \text { aizkora « hache » } \rightarrow \text { aizkol-begi « œil de la hache » }
\end{aligned}
$$

Il est bien connu que latérales et rhotiques partagent bon nombre de caractéristiques phonétiques et phonologiques, raison pour laquelle elles sont regroupées dans la classe naturelle des liquides. Premièrement, grâce à leur structure formantique claire proche de celle des voyelles, elles sont susceptibles de fonctionner comme noyau syllabique dans de nombreuses langues. Deuxièmement, en tant que consonnes à sonorité maximale, elles se réalisent librement dans des positions proches du noyau syllabique (comme dernier élément d'une attaque complexe ou premier élément d'une coda complexe). Troisièmement, latérales et rhotiques coronales sont articulées d'une manière semblable, ce qui détermine leur fonctionnement comme des variantes d'un même phonème dans des langues comme le japonais et le coréen.

Cela étant, le passage direct de /l/à /s/ se conçoit facilement. Comme les deux liquides coronales ont pratiquement le même lieu d'articulation (dans la zone alvéolaire), il suffit que la masse linguale se relève un peu pour gêner l'échappement latéral de l'air expiré et le rediriger audessus de la pointe de la langue. Cette transformation est aisément formalisable en phonologie, vu que [ \pm latéral] est le seul trait pertinent qui oppose les deux segments : 
(16)
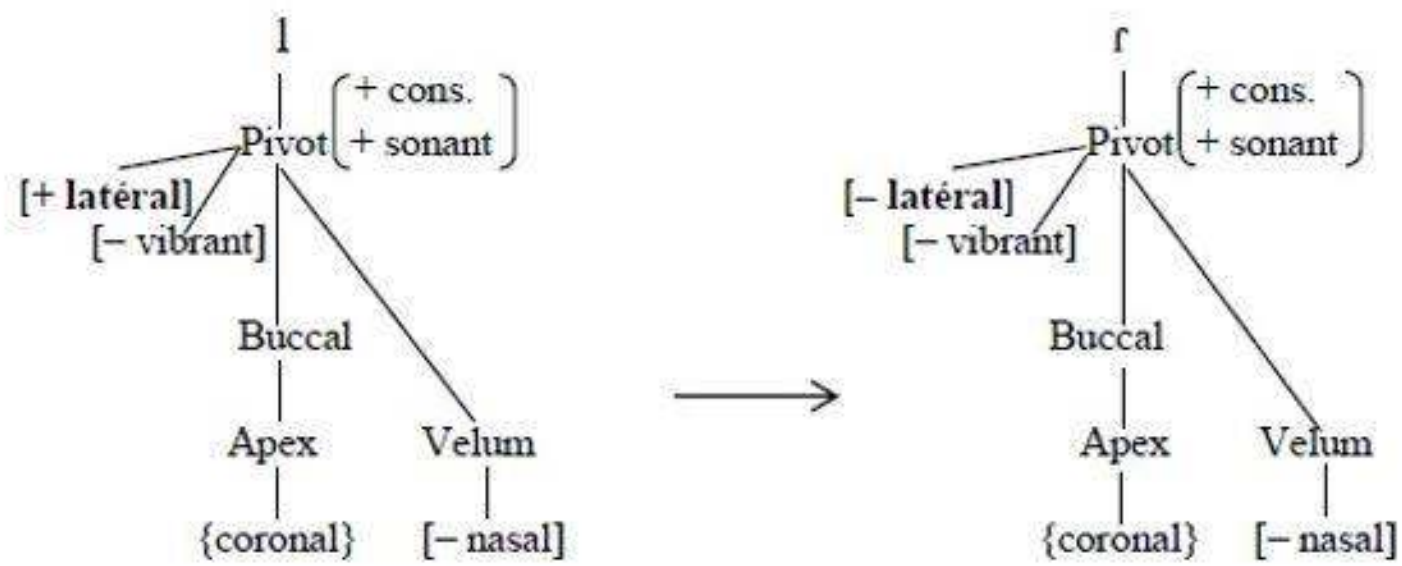

Un affaiblissement extrême du contact buccal peut entraîner l'amuïssement complet de la liquide, ce qui s'est effectivement produit en galicien-portugais au XII ${ }^{\mathrm{e}}$ siècle : VOLARE > voar « voler », CELLU > céu « ciel », SALUTE > saúde « santé » (Bourciez 1923 : 387). S'appuyant sur ces données, Baldinger $(1958: 252)$ conclut à une tendance articulatoire commune à deux zones géographiquement isolées ( $c f$. Carte 5) qui coïncident exactement avec les zones d'amuïssement $\mathrm{du} / \mathrm{n} /$ intervocalique ( $c f$. Carte 4$)$.

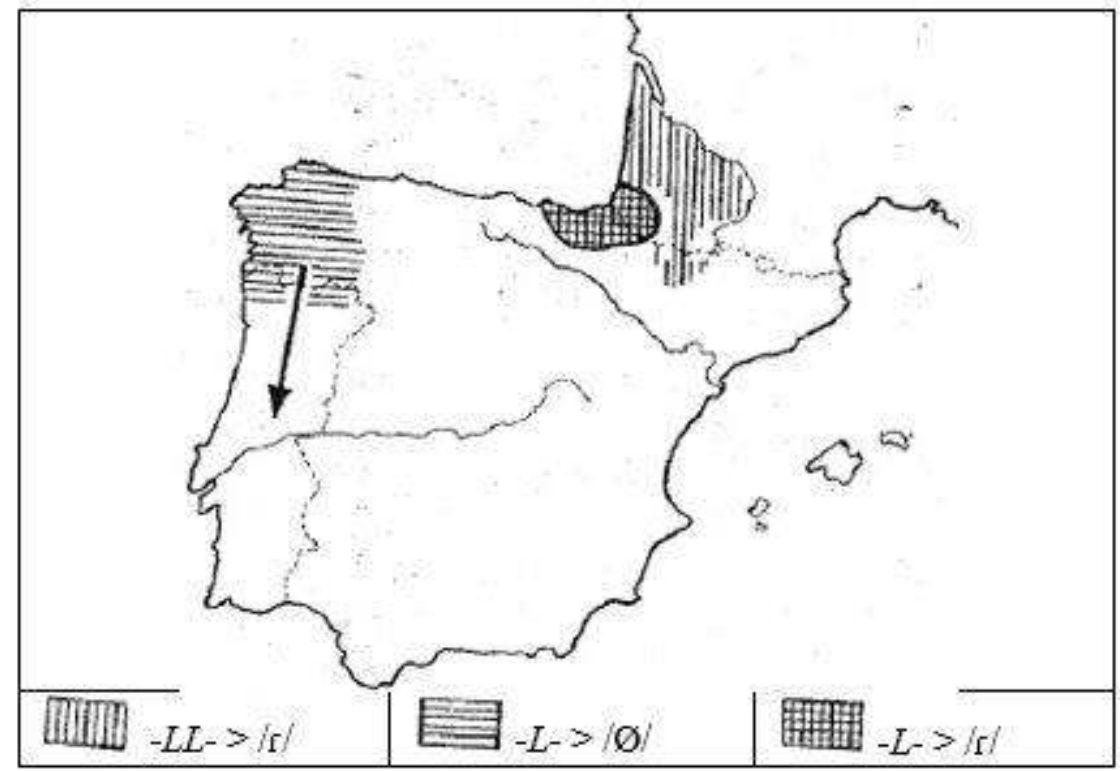

Carte 5 : Le traitement des latérales intervocaliques (Baldinger $1958: 252$ )

Cependant, le passage de $/ / /$ à $/ \mathrm{r} /$ semble ne pas avoir été direct en gascon. En témoigne, premièrement, le traitement différencié du - $L$ - intervocalique simple et du - $L L$ - double : BELLA $>/$ bero/ vs. PALA > /palo/. Deuxièmement, $-L L$ - devenu final après la chute d'une voyelle

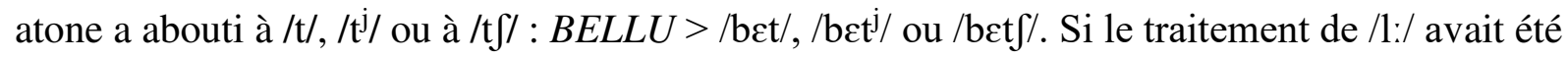
identique dans les deux contextes avant la chute des voyelles finales, ses produits ne devraient pas être si différenciés en finale et à l'intervocalique. Mais l'argument le plus solide contre le rhotacisme direct vient de divers parlers de l'Italie méridionale où celui-ci ne s'est opéré qu'à 
travers une phase intermédiaire rétroflexe (Rohlfs 1966 : 305-310, 328-334). Ainsi, on trouve en calabrais des réalisations comme $/ \mathrm{ka}^{\mathrm{l}} \mathrm{vallu} / \mathrm{et} / \mathrm{gal}$ l'ina/ pour CABALLU «cheval » et GALLINA. Rohlfs précise aussi que l'aboutissement le plus répandu de /1:/ est /d/ avec une concavité de la langue plus ou moins accusée selon les parlers calabrais, siciliens et salentins où il est attesté : /kalvadqu/, /lbedqu/ (<BELLU). Le changement s'étend jusqu'en Sardaigne et en Corse méridionale et dans la même zone du sud de l'Italie continentale /d/ peut subir ultérieurement un rhotacisme en passant à $/ \mathrm{r} /$ ou à $/ \mathrm{r} /$.

D'autre part, en Calabre septentrionale et plus fréquemment dans les zones gallo-italiennes de la Sicile, le rhotacisme peut affecter aussi le /l/ intervocalique simple :TELA >/tira/ «toile », $M E L E>/$ mere/ « miel », $A L A>/$ ara/ « aile ». A Novara, la rhotique issue de $-L$ - rejoint le sort de $-N$ - $(c f . \S 2.3)$ et s'élide comme dans la zone galégo-portugaise. Cela permet d'établir un parallèle avec certains dialectes basques qui connaissent une tendance plus ou moins accusée à faire tomber les « $r$ douces » entre voyelles (Gavel $1956: 4$ ). Ainsi, Coyos (1999: 76) signale qu'en souletin l'opposition entre rhotiques «douces » et «fortes » prend à l'intervocalique la forme /Ø/ /r/ : bera /'bea/ « lui-même » berra /'bera/ « le (la) même ». La même évolution n'est pas inconnue de l'Italie septentrionale non plus et se manifeste nettement en Ligurie qui, à en croire la description de Rohlfs (1966 : 306), a abouti dans les parlers montagnards à «una $\mathrm{r}$ poco energica, debolmente vibrata » complètement amuïe dans le reste du domaine : /tejra/, /ara/ > /teja/, /a/.

Prenant en compte les faits italiens, Bec (1968 : 93-94) suppose une phase rétroflexe également pour le gascon. Jusqu'à la chute des voyelles finales atones autres que /a/, celui-ci en était au stade /d/. A partir de ce moment-là, /d/ a connu un traitement différencié selon qu'il est resté intervocalique ou s'est retrouvé final. Dans le premier contexte il s'est affaibli en une rhotique alors que dans le deuxième il s'est renforcé par dévoisement ou par palatalisation/affrication :

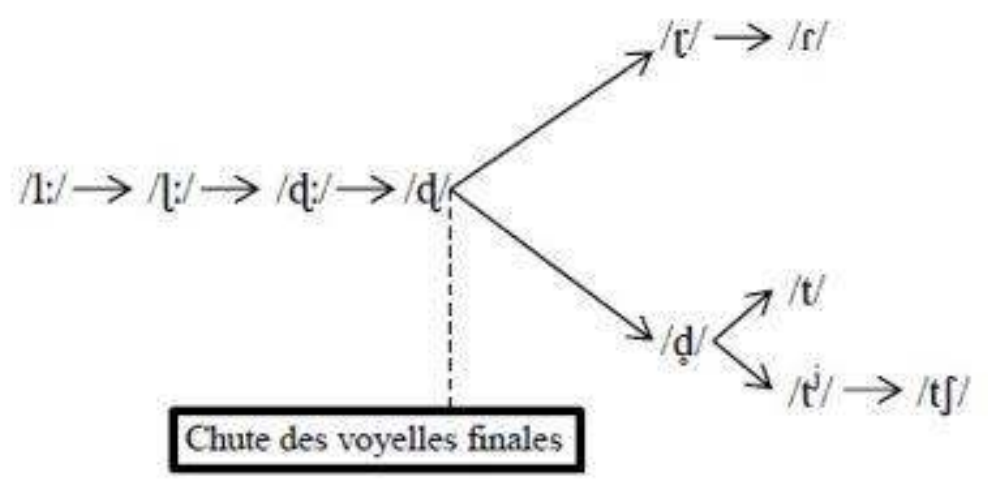

Pour ce qui est du - $L$ - simple, il est resté à l'abri de cette évolution sans doute à cause de son timbre vélaire (Bec 1968 : 95). Cela explique également pourquoi il s'est vocalisé en /w/ en finale : SALE > sau «sel », FILU > hiu « fil ».

En somme, l'affaiblissement de la latérale intervocalique (simple ou géminée) est loin de constituer une originalité de la seule zone basco-gasconne car il connaît une extension 
géographique remarquable dans de nombreuses variétés romanes. Il se manifeste sous des formes différentes en galicien-portugais, dans plusieurs zones de l'Italie continentale et

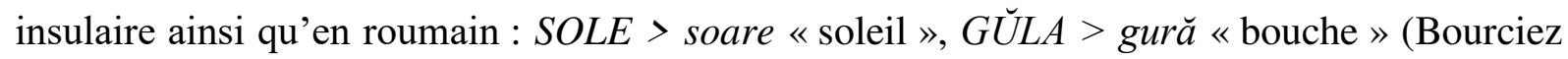
1923 : 165). Dans ce cas concret, les thèses substratistes paraissent d'autant moins probables que le gascon ne connaît pas le rhotacisme du /l/ intervocalique simple contrairement au basque. Ce dernier va même jusqu'à intervertir les aboutissements de $-L$ - et $-L L-: G U L A>$ gura « désir », CELLA > gela « chambre » (Allières 1992 : 807). Les changements examinés dans les deux dernières sous-sections semblent en revanche relever d'une tendance générale propre à certaines zones romanes à l'affaiblissement des sonantes coronales intervocaliques : chute de - $L$ - et $-N$ - en portugais, altération de $-L L$ - et chute de $-N$ - en Gascogne, chute de $-R$ - dans les Asturies et en Andalousie (Bourciez 1923 : 338), chute de $-L$ - et $-R$ - (primaire ou secondaire) en Ligurie $(P E R A>/$ pea/ « poire », $A L A>/ \mathrm{ara} />/ \mathrm{a} /)$, chute des trois sonantes coronales intervocaliques dans les aires gallo-italiennes de la Sicile, altération de - $L$ - et dialectalement de $-N$ - en roumain $(B E N E>/$ 'bire/ «bien »), de même qu'en istro-roumain (LUNA >/lure/) (Bourciez 1923 : 527).

\section{5. Le voisement des plosives sourdes après sonante}

Les deux derniers phénomènes qui seront examinés dans les lignes suivantes concernent le traitement des plosives $/ \mathrm{p} /, \mathrm{t} / \mathrm{et} / \mathrm{k} /$ dans deux contextes particuliers. Après nasale ou latérale tout d'abord, celles-ci demeurent généralement intactes en gascon (CANTARE > /kan'ta/ « chanter », BLANCA > /blanko/ «blanche », SALTARE > /saw'ta/ « sauter ») mais subissent un voisement dans une petite zone de la Gascogne pyrénéenne (vallées d'Aspe, d'Ossau et de Barétous) : /kanlda/, /sawlda/, //blango/ (Ravier 1991 : 87-88). Les seules variétés limitrophes qui connaissent le même changement sont le haut-aragonais et le basque, comme en témoigne le traitement que ce dernier a fait subir à ses emprunts au latin : ALTARE > aldare «autel », TEMPORA > denbora «temps », VOLUNTATE > borondate «volonté », GENTE > jende « gens », INCUDINE > ingude « enclume ». Orpustan (2009:7) est convaincu de l'ancienneté du phénomène qui serait directement attesté en ibère (bronze d'Ascoli). Toutefois, le roncalais et le souletin ne participent pas au changement et préservent les groupes originels intacts : ronc. altare, tenpra, borontate, gente ; soul. althá(r)e, bo(r)ontháte jénte, ünkhüde (Michelena 1954 : 149-150, Allières 1992 : 807, Coyos 1999 : 75).

A en croire Hualde (1991 : 136), dans les variétés basques qui connaissent la règle de voisement, celle-ci n'est plus active en synchronie que dans des processus de suffixation isolés où elle n'a pas lieu après une rhotique. Tel est le cas par exemple du suffixe perfectif -tu dont le comportement est illustré ci-dessous :

(18a) neka-tu «se fatiguer» har-tu «prendre » (18b) afal-du «dîner »

lan- $d u \ll$ enlever » 
A la recherche de la généralisation correcte qui capte le contexte d'application de la règle de voisement, Hualde affirme qu'elle ne prend effet qu'après les sonantes [- continu]. Sans exclure l'hypothèse que dans d'autres langues la latérale /1/ pourrait fonctionner comme une continue, l'auteur soutient qu'en basque elle se comporterait comme un segment [- continu] en vertu de cette même règle et d'une autre qui ne palatalise que les coronales non continues $/ \mathrm{n} /, / 1 /$, et variablement /t/ et /d/ (ibid. 104).

Cette analyse est cependant contredite par le fait que pour d'autres processus de la phonologie du basque la latérale fait bloc avec les rhotiques et les voyelles contre les nasales. Ainsi, les plosives sonores $/ \mathrm{b} /, / \mathrm{d} /$ et $/ \mathrm{g} /$ se spirantisent à l'intervocalique et après les liquides mais se réalisent occludées après les nasales. Il est toutefois à retenir le comportement du groupe [ld] où le basque a abouti (tout comme l'espagnol) à une réalisation discontinue par prolongement de l'occlusion coronale (ibid. : 100) :

\begin{tabular}{|c|c|c|c|}
\hline $\mathrm{N}, \mathrm{V}$ & I V & $r_{2} \mathrm{~V}$ & $\mathrm{~V}$ \\
\hline [embora] « tronc » & [alßoan] "à coté de » & [arßi] « navet » & [aßere] « bétail » \\
\hline [mendi] « montagne $»$ & [talde] « groupe » & [arøi] « mouton » & [aðar] « corne " \\
\hline [engo] " de là " & [alyoobi] « coton " & [aryi] « lumière » & [layun] «ami » \\
\hline
\end{tabular}

Nous croyons que le contenu phonétique des traits distinctifs en (9) est universel dans la mesure où il ne peut pas varier d'une langue à l'autre. Chaque segment répertorié dans l'API est donc défini par une même combinaison de traits dans n'importe quelle langue. Ces traits ont nécessairement la même valeur + ou - pour le segment en question mais n'ont pas la même pertinence pour les processus phonologiques des diverses langues.

Pour rendre compte du voisement des plosives après nasale et latérale en basque et en gascon, on peut envisager deux explications possibles. La première est purement phonologique et concerne la place occupée par les différents types de sonantes dans l'échelle de sonorité ( $c f$., entre autres, Farmer 1979, Schmid 1999, Montreuil 2000). A ce sujet, Burov (2012 : 267) soutient qu'il existe une échelle de sonorité minimale à trois termes seulement (obstruantes / sonantes / voyelles) et que c'est la seule universelle. Si certaines langues s'en tiennent à ce format minimal, d'autres peuvent opérer des subdivisions plus fines à l'intérieur de chacun des trois termes, en distinguant par exemple entre occlusives et fricatives ou entre sourdes et sonores dans le cadre des obstruantes, entre coronales et non coronales dans le cadre des fricatives, entre sifflantes et chuintantes dans le cadre des fricatives coronales, entre nasales, liquides et glides dans le cadre des sonantes, entre rhotiques et latérales dans le cadre des liquides $^{20}$, etc. Si l'on envisage donc l'échelle de sonorité dans une extension plus large en rapport avec les faits basco-gascons, elle devrait avoir la forme suivante :

20 Labrune (2005) soutient que les rhotiques sont universellement plus sonores que les latérales. 


\section{Echelle de sonorité}

$\begin{array}{ll}7^{\circ} & \text { voyelles } \\ 6^{\circ} & \text { semi-voyelles } \\ 5^{\circ} & \text { rhotiques } \\ 4^{\circ} & \text { latérales } \\ 3^{\circ} & \text { nasales } \\ 2^{\circ} & \text { fricatives } \\ 1^{\circ} & \text { plosives }\end{array}$

Comme les latérales et les nasales sont placées moins haut que les rhotiques dans cette hiérarchie, elles restent plus près des plosives ou en sont plutôt moins éloignées. En effet, dans plusieurs langues, et en cas d'assimilation surtout, deux segments ne peuvent interagir que s'ils sont suffisamment similaires. Dans cet ordre d'idées, si l'on considère les exemples en (18), on constate qu'à partir d'un certain degré de sonorité ou à partir d'un certain écart de sonorité entre Rime et Attaque l'assimilation de voisement ne se produit pas. La consonne initiale du suffixe -tu n'interagit ni avec les voyelles, ni avec les rhotiques $\left(\geq 5^{\circ}\right)$, mais uniquement avec les segments de sonorité plus basse $\left(\leq 4^{\circ}\right)$. Ces faits rejoignent également la contrainte imposée par les deux langues à leurs séquences d'obstruantes $\left(1^{\circ}-2^{\circ}\right)$ qui doivent s'accorder elles aussi sur la valeur du trait [voisé].

L'autre explication possible du voisement des plosives après nasale et latérale se trouve à l'interface phonétique/phonologie. En effet, l'observation de Hualde que /1/ pourrait fonctionner comme [- continu] dans certaines langues et comme [ + continu] dans d'autres tient à ce que son articulation combine un contact complet entre la pointe de la langue et la zone alvéodentale avec un libre passage de l'air expiré des deux côtés de la masse linguale. C'est la raison pour laquelle nous avons opté en (9) pour la scission du trait traditionnel [ \pm continu] en deux : [ \pm flux continu] (ou [ \pm continu] tout court) et [ \pm occlusion buccale] (représenté comme [ \pm occludé]). Cela étant /l/sera défini comme [+ continu, + occludé] et s'opposera à $/ \mathrm{r} / \mathrm{et} / \mathrm{r} /$ qui auront les spécifications [+ continu, - occludé]. En réalité, les deux rhotiques impliquent un passage de l'air au-dessus de la pointe de la langue qui ne fait que vibrer (plus ou moins intensément) contre la zone alvéodentale sans établir de contact prolongé. Cette entrave minimale au flux d'air explique pourquoi les rhotiques sont relativement plus sonores que les latérales: dans les langues du monde, les premières forment plus facilement des noyaux syllabiques et des attaques complexes avec des obstruantes que les secondes.

Bref, d'après ce qui a été dit, les plosives se prêtent mieux à une assimilation de voisement avec les latérales et les nasales qu'avec les rhotiques du fait qu'elles partagent avec les deux premières classes naturelles de consonnes un plus grand nombre de traits communs, ce qui revient à dire qu'elles y sont plus similaires : 
(21)
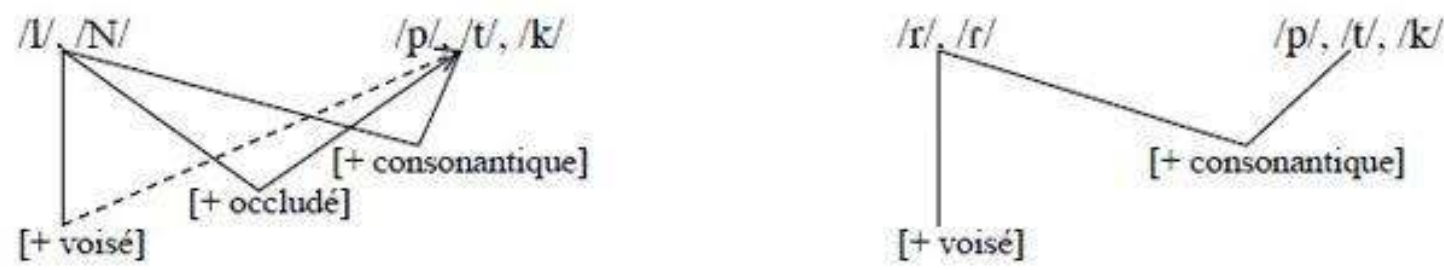

Le même type de sonorisation est attesté dans certains dialectes du sud et du centre de l'Italie (ombrien, apulien, abruzzais, napolitain, calabrais, campanais, etc.), à ceci près que dans certaines de ces zones (Pouilles, Campanie, Marches, Abruzzes) on l'observe également après rhotique : SPIR(I)TU > /'spirdə/ « esprit», VER(I)TATE > /erldate/ « vérité », ALTU > /laldo/ « haut », BLANCU > /bjango/ «blanc », DENTE > /dende/ « dent », ANTICU > /an'dikə/ « antique » (Rohlfs $1966: 347,363,375)$. Allières (1992: 808) signale d'ailleurs une tendance ancienne au voisement après $r$ également pour le haut-aragonais : (EX)URTICA > (ch)ordigal/$c a$ « ortie », LACERTU (avec divers croisements) > sagard(i)ana « petit lézard ». Il semble que l'Aquitaine a connu elle aussi à une date lointaine un voisement après l'ensemble des sonantes, comme en témoigne l'anthroponyme antique Inderca (nom de femme en Aquitaine) à côté d'Andergus, ainsi que certaines formes isolées comme pord «port », retrouvées par Baldinger (1958 : 257) dans des manuscrits médiévaux. Si c'est effectivement le cas, le processus pourrait n'avoir restreint qu'ultérieurement son domaine d'application. Mais avant de tirer des conclusions sur la similitude des faits basco-béarno-aragonais avec ceux de l'Italie centroméridionale, il paraît indispensable d'examiner le comportement des plosives sourdes dans un autre contexte particulier.

\section{6. La conservation des plosives sourdes intervocaliques}

La sonorisation des plosives après sonante est d'autant plus inhabituelle que dans la zone bascopyrénéenne déjà évoquée elle n'a pas eu lieu entre voyelles, position d'affaiblissement par excellence. En effet, si dans la plupart du domaine gascon $/ \mathrm{p} / \mathrm{t} / \mathrm{t} / \mathrm{et} / \mathrm{k} /$ intervocaliques passent aux sonores correspondantes (ARTICA > /arltigo/ «terrain défriché », APICULA > /albe $\mathrm{lo} /$ « abeille », MUTARE > /mylda/ «muer ») ${ }^{21}$, dans les vallées d'Aspe, d'Ossau et de Barétous, celles-ci ont été préservées intactes : /arltiko/, /alpe אo/, /mylta/. Ravier (1991 : 87-88) et Allières (1992 : 804), à qui nous devons ces exemples, mettent le conservatisme de cette petite aire béarnaise en rapport avec les faits de l'aragonais $(A C U T U>/ \mathrm{a}$ kuto/ «aigu », LEPORE > /ljepre/ ou /ljapre/ « lièvre ») et du basque dont les emprunts au latin montrent la même résistance des plosives intervocaliques au voisement (PACE > bake «paix », PICE > bike « poix », VOLUNTATE > borondate, RIPA > erripa « rive », ROTA > errota).

21 C'est d'ailleurs le traitement normal des plosives sourdes intervocaliques dans tout le domaine occitan. 
On sait que, d'une manière générale, l'affaiblissement des plosives sourdes intervocaliques est la marque de l'ibéro-, du rhéto- et du gallo-roman (y compris des dialectes gallo-italiens septentrionaux et siciliens), alors que leur conservation est caractéristique de l'italien centroméridional $^{22}$ et du balkano-roman (y compris du dalmate déjà disparu). Glessgen (2007 : 157) va même jusqu'à opposer une «Romania occidentale (innovatrice)» à une «Romania orientale (conservatrice) » sur le traitement des plosives sourdes intervocaliques qui serait une des grandes lignes de démarcation entre les deux complexus. Cela étant, cette étroite zone constituée, grosso modo, par le béarnais, le haut-aragonais et le basque représente un îlot de conservatisme au sein de territoires connaissant une évolution phonétique plus poussée. Bourciez (1923 : 162) attribue le caractère archaïsant de cette zone à l'influence de la "phonétique euskarienne », alors que Jungemann (1956) à l'absence de véritable influence celtique et de bilinguisme gaulois-latin. Mais il serait peut-être un peu plus prudent d'affirmer que la zone pyrénéenne en question est restée à l'abri d'influences celtibères. Il est bien connu en effet que le relief montagneux favorise la sédentarité des populations, l'isolement dialectal et la fixation d'un état de langue plus ancien. Les vastes plaines, en revanche, facilitent la circulation et la mobilité des personnes, accélérant ainsi la diffusion des changements linguistiques.

En $\S 2.5$, le voisement des plosives après sonante nasale et latérale a été mis sur le compte de la relative similarité des consonnes en interaction, alors que l'absence de voisement dans la même zone après rhotique et à l'intervocalique a été attribuée à la dissimilarité des articulations en contact. Cette dissimilarité se traduit par l'absence d'occlusion dans les articulations vocaliques et rhotiques qui leur fait occuper des paliers trop élevés dans l'échelle de sonorité. Néanmoins, ce double traitement des plosives sourdes selon le contexte a été qualifié d'inhabituel : elles se maintiennent intactes en position faible (V_V) mais s'affaiblissent en position forte $\left(\mathrm{C}_{-} \mathrm{V}\right)$. Il est à retenir en effet que le reste du domaine gascon en opère un traitement tout à fait inverse : CANTARE $>/ \mathrm{kan}^{\mathrm{l}} \mathrm{ta} /$, MUTARE $>/ \mathrm{my}^{\mathrm{l}} \mathrm{\partial a} /{ }^{23}$. Baldinger (1958 : 257) est d'avis que pour cet état de fait «l'influence indigène semble être hors de doute » et cite Gamillscheg qui y voit un substrat vascon. Orpustan (2009: 7), lui, signale que le voisement après sonante est directement attesté dans des monuments écrits ibères où l'on constate aussi une "légère prédominance » des sonores sur les sourdes à l'initiale ( $c f$. note 24). Même si nous avons retrouvé la même distribution des plosives (sourdes à l'intervocalique et

22 L'Italie centro-méridionale montre toutefois certaines hésitations et l'on y trouve un nombre considérable d'exceptions à la tendance à conserver intacts /p/, /t/ et /k/ intervocaliques (Rohlfs 1966).

23 La situation est d'autant plus étrange que le basque a affaibli en sonores les plosives latines également en début de mot, position forte par excellence : PACE > bake, TEMPORA > denbora, CERESIA > gerezi «cerise». On peut être tenté de voir dans tout cela une sorte d'inversion des positions fortes et faibles. A titre d'explication, Martinet $(1955,1981)$ reconstruit un proto-système du basque qui opposerait des « sourdes fortes » ou aspirées à des « sourdes douces $»: / \mathrm{p}^{\mathrm{h}} / \sim / \mathrm{b} /, / \mathrm{t}^{\mathrm{h}} / \sim / \mathrm{d} /, / \mathrm{k}^{\mathrm{h}} / \sim / \mathrm{g} /$. D'après lui, les plosives non aspirées du latin auraient été ramenées en basque au type doux, d'où le voisement en début de mot. 
sonores après sonante) dans l'Italie centro-méridionale, ce traitement apparaît comme le moins étendu géographiquement en comparaison des autres. Il n'est donc nullement invraisemblable que, dans son extension occidentale au moins, il soit dû à un contact de langues et à l'archaïsme en puissance de la zone concernée.

\section{Observations conclusives}

Un aperçu général des changements examinés dans la section précédente fait apparaître que ceux-ci représentent soit des phénomènes d'affaiblissement, soit des phénomènes de renforcement. Il en est ainsi car la faible perception d'une combinaison de traits, d'un segment dans un contexte particulier ou d'une structure syllabique marquée peut induire des évolutions dans des sens opposés : un affaiblissement ultérieur ou un renforcement. Dans la première catégorie seront classés l'aspiration de $/ \Phi /$, le traitement de $/ \mathrm{n} /$ et des latérales intervocaliques, ainsi que le voisement des plosives après sonante nasale et latérale ; quant à la seconde, elle englobe la prothèse devant le /r:/ initial renforcé, le passage de $/ \phi$ / à une plosive labiale en basque et la conservation des $/ \mathrm{p} / \mathrm{/} / \mathrm{t} / \mathrm{et} / \mathrm{k} /$ intervocaliques.

Ces changements ont fait l'objet du présent article parce que selon beaucoup de linguistes ils sont censés constituer une spécificité basco-gasconne et refléter des tendances articulatoires communes aux deux langues. Ces affirmations ont logiquement donné lieu à des thèses substratistes, ce qui nous a conduit à examiner également les conditions historiques de diffusion du latin dans la zone en question. Les éléments d'étude aréologique introduits en $\S 2$ montrent cependant qu'aucun des changements examinés n'est limité à la région basco-aquitaine. Qui plus est, ils se retrouvent tous, sans exception, loin au-delà de la Péninsule ibérique et de l'Aquitaine dans d'autres variétés romanes.

Plus concrètement, la prothèse devant le $r$ initial est attestée aussi en macédo-roumain, dans le domaine rhétique et en émilien ; l'aspiration de $/ \phi$ / se retrouve dans le sud et le nord de l'Italie ; l'amuïssement $\mathrm{du} / \mathrm{n} /$ intervocalique apparaît également en gallo-italien; la rétroflexion, le rhotacisme ou la chute des latérales $-L$ - et $-L L$ - intervocaliques est documentée en Italie méridionale (continentale et insulaire) et en roumain ; la sonorisation des plosives après sonante est typique de l'Italie centro-méridionale ; la conservation des plosives sourdes intervocaliques est une caractéristique générale de l'Italie centro-méridionale et du balkano-roman.

On peut constater aussi que le basque et le gascon partagent cinq de ces traitements avec diverses variétés d'italien ( $§ 2.2 / 3 / 4 / 5 / 6)$, trois avec l'aragonais ( $\$ 2.1 / 5 / 6)$, deux avec le galicien-portugais $(\S 2.3 / 4)$, deux avec le roumain $(\S 2.4 / 6)$, un avec le castillan $(\S 2.2)$, l'engadinois $(\S 2.1)$ et le macédo-roumain $(\S 2.1)$. On ne peut s'empêcher de remarquer en outre qu'aucun de ces traitements n'est caractéristique du gallo-roman (septentrional ou 
méridional $)^{24}$, ce qui confirme la place particulière qu'occupe le gascon parmi les dialectes occitans.

Or, le fait que les phénomènes étudiés en $§ 2$ connaissent une extension géographique considérable à travers la Romania ne peut pas invalider complètement les théories substratistes. Leur analyse montre seulement que tout changement phonologique, qu'il ait été conditionné extérieurement ou pas, a nécessairement une motivation interne (phonétique et/ou phonologique). Dans cet ordre d'idées, un changement phonologique est d'autant plus répandu et peut surgir d'autant plus souvent qu'il est naturel. Ainsi, la chute des consonnes nasales (avec ou sans nasalisation compensatoire), le rhotacisme des consonnes coronales, l'aspiration des fricatives ou l'épenthèse dans des clusters consonantiques sont des changements translinguistiques parce qu'ils répondent à des contraintes naturelles d'articulation et/ou de perception. Il est clair par exemple que le traitement de $/ \phi /$ ou du $/ \mathrm{r} /$ initial dans de vastes zones de la Péninsule ibérique et en Aquitaine reflète une même tendance articulatoire sous-jacente largement diffusée à une époque lointaine, et que le traitement des plosives sourdes originelles dans une zone basco-béarno-aragonaise beaucoup plus étroite est un phénomène de contact de langues dans un terrain orographique favorisant l'isolement et le conservatisme. Il faut cependant se garder de confondre contact de langues (général) avec influences substratiques (particulier) car il n'est rien de plus imprudent que de chercher la part que les Ibères, les Aquitains, les Vascons ou d'autres auraient eu dans ces tendances évolutives.

\section{Références}

Allières, Jacques. 1992. Gascón y euskera : afinidades e interrelaciones lingüísticas. Anuario del Seminario de Filología Vasca « Julio de Urquijo » XXVI-3. 801-812.

Baldinger, Kurt. 1958. La position du gascon entre la Galloromania et l'Ibéroromania. Revue de linguistique romane 22. 243-292.

Barnes, Jonathan Allen. 2002. Positional neutralization: a phonologization approach to typological patterns. Ph.D. dissertation, University of California, Berkeley.

Bec, Pierre. 1968. Les interférences linguistiques entre gascon et languedocien dans les parlers du Comminges et du Couserans. Paris : PUF.

Bec, Pierre. 1995. La langue occitane. Paris : PUF, 6 édition corrigée.

24 D'autres changements, comme l'apparition de /y/ et de voyelles nasales en souletin et en roncalais, qui n'ont pas été étudiés ici parce que de moindre importance et plus récents, montrent toutefois des influences du français sur le basque de France. 
Bec, Pierre. 1999. Encore sur la prononciation du $u$ en occitan médiéval : l'apport de l'ancien gascon au problème. Revue des langues romanes III.2. 213-222.

Bourciez, Édouard Eugène Joseph. 1923. Eléments de linguistique romane, Paris : Klincksieck.

Bourciez, Édouard Eugène Joseph. 1936. Le domaine gascon. Revue de linguistique romane, XII. 1-9.

Burov, Ivaylo. 2012. A propos de la syllabation des groupes consonantiques romans $/ \mathrm{sC} /$. Annuaire de l'Université de Sofia Saint Clément d'Ohrid 105. 239-279.

Burov, Ivaylo. 2013a. Les phénomènes de sandhi dans l'espace gallo-roman, Sofia : Presses universitaires St. Clément d'Ohrid.

Burov, Ivaylo. 2013b. Fonetica e fonologia dei nessi romanzi /s/ + consonante », Actes $d u X^{e}$ colloque des doctorants et des enseignants non habilités de la Faculté des Lettres classiques et modernes, Sofia : Presses universitaires St. Clément d'Ohrid. 102-109.

Cavalli-Sforza, Luca \& Francesco Cavalli-Sforza. 1994. Qui sommes-nous? Paris : Flammarion.

Chomsky, Noam \& Morris Halle. 1973. The sound pattern of English (1968), traduit partiellement en français par P. Encrevé sous le titre de Principes de phonologie générative, Paris : Seuil.

Clements, George N. 1985. The geometry of phonological features. Phonology Yearbook 2. 225-252.

Clements, George N. 1993. Lieu d'articulation des consonnes et des voyelles. In Bernard Laks \& Annie Rialland (éds.), Architecture des représentations phonologiques, 101-145. Paris : Editions du CNRS.

Clements, George N. \& Elizabeth V. Hume. 1995. The internal organization of speech sounds. In John A. Goldsmith (éd.), Handbook of Phonological Theory, 245-306. Oxford : Blackwell.

Coyos, Jean-Baptiste. 1999. Le parler basque souletin des Arbailles. Une approche de l'ergativité. Paris : L'Harmattan.

Encrevé, Pierre. 1988. La liaison avec et sans enchaînement. Phonologie tridimensionnelle et usages du français. Paris : Les Editions du Seuil.

Farmer, Ann. 1979. Phonological markedness and the sonority hierarchy. In Kenneth J. Safir (éd.), MIT working papers in linguistics 1 : Papers on syllable structure, metrical structure and harmony processes, 172-177. Cambridge : MIT. 
Gavel, Henri. 1951. Grammaire historique élémentaire de la langue espagnole. ParisToulouse : Prival-Didier.

Gavel, Henri. 1956. Note sur des chutes de labiale initiale devant voyelle vélaire en basque. Via Domitia III. Annales publiées par la Faculté des Lettres de Toulouse, Année 5, Fascicule 4. 15 .

Glessen, Martin-Dietrich. 2007. Linguistique romane. Domaines et méthodes en linguistique française et romane. Paris : Armand Colin.

Goldsmith, John A. 1979. Autosegmental phonology. New York : Garland Press.

Halle, Morris, Bert Vaux \& Andrew Wolfe. 2000. On feature spreading and the representation of place of articulation. Linguistic Inquiry 31.3. 387-444.

Hirschfeld, Otto. 1913. Aquitanien in der Römerzeit. Kleine Schriften, Berlin, 209-238.

Hualde, José Ignacio. 1991. Basque Phonology. London : Rutledge.

Hubschmid, Johannes. 1954. Pyrenäenwörter vorromanischen Ursprungs und das vorromanische Substrat der Alpen. Acta salamanticensia, VII, Salamanca.

Jungemann, Fredrick Henry. 1956. La teoría del sustrato y los dialectos hispano-romances y gascones, Madrid : Gredos.

Kaye, Jonathan \& John Lowenstamm. 1984. De la syllabicité. In François Dell, Daniel Hirst \& Jean-Roger Vergnaud (éds.), Forme sonore du langage. Paris : Hermann. 123-159.

Labrune, Laurence. 2005. Autour de la syllabe: les constituants prosodiques mineurs en phonologie. In Noël Nguyen et al. (éds), Phonologie et phonétique : forme et substance, 95116. Paris : Hermès.

Lafont, Robert. 1991. L'occitan. Histoire interne de la langue II. Lexique. In Günter Holtus et al. (eds.), Lexikon der Romanistischen Linguistik, V/2, 18-23.Tübingen: Max Niemeyer Verlag.

Martinet, André. 1955. Economie des changements phonétiques. Traité de phonologie diachronique. Berne : Francke.

Martinet, André. 1981. Phonologie synchronique et diachronique du basque. In Euskaltzaindia (éd.), Iker 1 : Euskalarien Nazioarteko jardunaldiak, 59-74. Bilbo : Euskaltzaindia.

Michelena, Luis. 1954. La posición fonética des dialecto vasco del Roncal. Via Domitia I. Annales publiées par la Faculté des Lettres de Toulouse, Année 3, Fascicule 4. 123-157. 
Michelena, Luis. 1990. Fonética histórica vasca. San Sebastián: Diputación Foral de Guipúzcoa (Primera edición : 1961).

Milalrdet, Georges. 1910. Etudes de dialectologie landaise. Toulouse : É. Privat.

Montreuil, Jean-Pierre Y. 2000. Sonority and derived clusters in Raeto-Romance and GalloItalic. In Lori Repetti (éd.), Phonological theory and the dialects of Italy, 211-237. Amsterdam/Philadelphia : John Benjamins.

Orpustan, Jean-Baptiste. 2009. L'ibère et le basque : recherches et comparaisons. HAL archives ouvertes. fr (http://artxiker.ccsd.cnrs.fr/artxibo-00000017).

Oyharçabal, Bernard. 2005. Etudes des populations et singularité linguistique du Pays basque. HAL archives ouvertes. fr (http://artxiker.ccsd.cnrs.fr/artxibo-00465824).

Ravier, Xavier. 1991. Les aires linguistiques. Lexikon der Romanistischen Linguistik, In Günter Holtus et al. (eds.), Lexikon der Romanistischen Linguistik, V/2, 80-105. Tübingen : Max Niemeyer Verlag.

Rohlfs, Gerhard. 1952. Sur une couche préromane dans la toponymie de la Gascogne et de 1'Espagne du Nord. Revue de philologie espagnole XXXVI. 209-256.

Rohlfs, Gerhard. 1966. Grammatica storica della lingua italiana e dei suoi dialetti (Fonetica). Torino : Einaudi.

Ronjat, Jules. 1930. Grammaire historique des parlers provençaux modernes, vol. II, Montpellier : Société des langues romanes.

Sagey, Elizabeth C. 1986. The representation of features and relations in nonlinear phonology. PhD. dissertation, Cambridge, Massachusetts.

Schmid, Stephan. 1999. Fonetica e fonologia dell'italiano. Torino : Paravia.

Tuaillon, Gaston. 1976. Comportements de recherche en dialectologie française. Paris : CNRS.

Videgain, Charles. 2003. La langue basque ou euskara : incertitudes et faits avérés. Clio. URL :http://www.clio.fr/bibliotheque/la_langue_basque_ou_euskara_incertitudes_et_faits_av eres.asp 